\title{
Quotient completion for the foundation of constructive mathematics
}

\author{
Maria Emilia Maietti and Giuseppe Rosolini
}

\begin{abstract}
We apply some tools developed in categorical logic to give an abstract description of constructions used to formalize constructive mathematics in foundations based on intensional type theory. The key concept we employ is that of a Lawvere hyperdoctrine for which we describe a notion of quotient completion. That notion includes the exact completion on a category with weak finite limits as an instance as well as examples from type theory that fall apart from this.
\end{abstract}

Mathematics Subject Classification (2010). 03G30 03B15 18C50 03B20 03F55.

Keywords. Quotient completion, split fibration, type theory, setoid.

\section{Introduction}

Category theory provides a language to investigate the syntax and the semantics of formal systems on the same ground, as it provides an appropriate abstraction useful to bring to the foreground an algebraic structure that usually remains hidden behind both. In fact, the present paper is a plain example of how category theory offers a language which is suitable to describe a key property that foundations of constructive mathematics should have according to 23 .

In the following part of the Introduction, we address the relevance of such abstract properties. After that we describe the category-theoretic concepts that are dealt with in the paper. Finally, we examine two quotient models based on intensional type theory.

The need of quotient completion to found constructive mathematics. There are various foundations for constructive mathematics available in the literature: some are formulated in axiomatic set theory, others in category theory, yet others in type theory. In fact there is no foundation for constructive mathematics as standard as the theory ZFC is for classical mathematics.

Project MIUR-PRIN McTAFI provided support for the research presented in the paper. 
The authors of 23. propose to look for a minimalist foundation which could form a common core for the most relevant constructive theories. Its finalized construction is in 21].

In loc.cit. the authors also state that a foundation for constructive mathematics should make it evident which key aspects differentiate it from classical mathematics. For instance, contrary to classical proofs, constructive proofs enjoy the existence property, i.e. one can extract programs that compute witnesses of existential statements occurring in them. Even more, any proof of a constructive system should be seen as a program. Hence, ideally, a foundation for constructive mathematics should be at the same time a set theory, in which to formalize mathematical theorems, and a programming language in which to extract the computational contents of mathematical proofs.

Type theory provides examples of such formal systems, such as MartinLöf's Intensional Type Theory 25, or Coquand's Calculus of Constructions 8. But there is a problem in adopting such type theories as a foundation for constructive mathematics. First of all they do not validate extensional features used in the everyday practice of mathematics such as extensional equality of sets or of functions, nor do they offer quotient constructions. Indeed, if one wants that these systems act as useful functional programming languages, they must meet decidability issues on typing of proofs which are incompatible with extensional features. This is argued in more formal terms (with the notion of proofs-as-programs theory) in [23].

The solution adopted in practice in the formalization of mathematics in type theory is to represent extensional concepts in a model built on top of the type theory, for example by using setoids, see [12, 1]. A main drawback of this approach for a constructive mathematician is that working with setoids and especially with dependent setoids - is extremely complicated compared to adopting a foundation in the same vein as ZFC for classical mathematics, like for instance Aczel's CZF or Friedman's IZF. A natural solution is to work with an axiomatization of the quotient model of setoids supported by the intensional type theory instead of working directly in the model. In a sense, someone who would like to have a foundation for constructive mathematics based on a type theory is naturally led to abandon the traditional view of having a unique system to formalize mathematics in favour of a two-level foundation where one level is used for program extraction and the other to formalize mathematics.

This is the central idea of the notion of constructive foundation put forward in [23. There it is required that a foundation for constructive mathematics should be a two-level theory: one level, named intensional, should be used as a programming language; the other, which is called extensional, should be closed under standard extensional constructs in order to be used as the actual formal system in which to perform mathematical proofs, and it should be seen as an abstraction of the intensional level according to Sambin's forget-restore principle, see [28. In 21] it was stated that to satisfy the link between the two levels in [23] it is enough to interpret the extensional 
level in the intensional one by means of a quotient completion of the latter, i.e. to see the extensional level as a fragment of the internal language of a quotient completion built on the intensional one. The two-level minimalist foundation in 21] provides an example of such a constructive foundation.

What remains to specify in the notion of constructive foundation in 21 is what one means "abstractly" by quotient completion.

In particular one wants to see whether the construction performed in 21] - the quotient model built over the intensional level to interpret the extensional level - is an instance of a free construction on categories with structure.

In the literature on category theory various constructions of quotient completion have been studied, for example in [7, 2, 6, These constructions all rely on defining quotients as stable effective coequalizers of monic equivalence relations. Hence, they all produce exact categories - and indeed they usually go under the name of exact completions.

But, as we observe in the present paper, the construction of quotients adopted in 21] does not necessarily lead to an exact category and therefore it cannot be an exact completion. This motivates the quest for a more general notion of quotient completion than the exact completion.

In this paper we accomplish this task by relativizing the notion of quotient to that of a suitable hyperdoctrine: the fibers act as the "logic" in which to consider equivalence relations. With respect to those, we introduce a notion of quotient in the base category of the hyperdoctrine, and we prove that that notion is algebraic. In other words, there is a universal construction that "freely adds" quotients for the equivalence relations without adding any further power to the logic.

We use a weakened notion of Lawvere hyperdoctrine [16, 18, 17, 19], here simply called "elementary doctrine", with respect to which we present a universal construction of quotient completion, which we call "elementary quotient completion".

Instances of this construction include both the quotient model in [21] and the exact completion of a category with finite limits in [5]. Indeed, the study of the elementary quotient completion helps to isolate, in the doctrine setting, the properties of a model "with quotients" and to handle those properties independently from one another. Thanks to the more general setting than that, say, of categories with (weak) finite limits, it is also possible to analyse the properties of the elementary quotient completion that are similar to the exact completion, such as closure under exponentials in [6].

As a biproduct of all this, we also obtain a clear explanation of the well-known result that the category of total setoids à la Bishop built over Martin-Löf's type theory is the exact completion of an appropriate category with weak finite limits, see [26, 4, 2]. 


\section{Doctrines}

We introduce the notion of doctrine that will be used to specify that of quotient. This notion is an obvious generalization of that of a hyperdoctrine. Hyperdoctrines were introduced, in a series of seminal papers, by F.W. Lawvere to synthesize the structural properties of logical systems, see [16, 18, 17, 19]. His crucial intuition was to consider logical languages and theories as indexed categories and to study their 2-categorical properties. For instance, connectives and quantifiers are determined by adjunctions. That approach proved to be extremely fruitful, see [24, 15, 14, 30, 31] and the references therein.

Recall from 16] that a hyperdoctrine is a functor $F: \mathscr{C}^{\mathrm{op}} \longrightarrow$ Heyt from a cartesian closed category $\mathscr{C}$ to the category of Heyting algebras satisfying some further conditions: for every arrow $f: A \rightarrow B$ in $\mathscr{C}$, the homomorphism $F_{f}: F(B) \rightarrow F(A)$ of Heyting algebras $-F_{f}$ denotes the action of the functor $F$ on the arrow $f$-has a left adjoint $\mathbb{H}_{f}$ and a right adjoint $V_{f}$ satisfying the Beck-Chevalley condition.

The intuition is that a hyperdoctrine determines an appropriate categorical structure to abstract both notions of first order theory and of interpretation.

A many-sorted first order theory gives rises directly to a hyperdoctrine $F: \mathscr{C}^{\mathrm{op}} \longrightarrow$ Heyt - a detailed presentation is given as example 2.2.

- the objects of $\mathscr{C}$ are declarations of sort variables

- a morphism $f: A \rightarrow B$ of $\mathscr{C}$ is a list of terms of the sorts in $B$, in the variables in the declaration $A$

- an object $P$ in $F(A)$ is a property written with the variables declared in $A$

- a morphism $P \leq Q$ in $F(A)$ shows that property $Q$ follows from the property $P$

- a functor $F_{f}: F(B) \rightarrow F(A)$ represents the substitution, in properties of the sort $B$, of the terms $f$ for the variables in $B$

- the adjoints $\mathcal{H}_{f}$ and $\forall_{f}$ represent the existential quantifier and the universal quantifier, respectively.

Another instance of hyperdoctrine $F: \mathscr{C}^{\mathrm{op}} \longrightarrow$ Heyt is the following:

- the objects of $\mathscr{C}$ are sets

- a morphism $f: A \rightarrow B$ of $\mathscr{C}$ is a function into the set $B$ from the set $A$

- an object $P$ in $F(A)$ is a subset of the set $A$

- a morphism $P \leq Q$ in $F(A)$ indicates that $Q$ contains $P$

- a functor $F_{f}: F(B) \rightarrow F(A)$ acts by inverse image along $f$ on subsets of the set $B$

- the adjoints $\exists_{f}$ and $V_{f}$ must be evaluated, on a subset $P$ of $A$, respectively as $\mathcal{G}_{f}(P)=\{b \in B \mid \exists a \in A[b=f(a) \wedge a \in P]\}$

Thus a model of the many-sorted first order theory determines precisely a functor from the former hyperdoctrine to the latter, as already pointed out in [16, 18]. 
Our aim is to take advantage of the algebraic presentation of logic offered by hyperdoctrines and we shall consider a more general notion with the structure needed to define a quotient of an equivalence relation from that perspective. We shall follow [18] and use the word "doctrine" with some attribute to christen the more general notion and others derived from it. We shall be able to separate the logical components producing a universal construction of completion by quotients of doctrines.

Somehow reflecting the essential logical structure that is needed in order to present the theory of an equivalence relation, the basic concept is that of a contravariant functor from a category with finite products into the category of inf-semilattices and inf-preserving maps.

Definition 2.1. A primary doctrine is a functor $P: \mathscr{C}^{\mathrm{op}} \longrightarrow \operatorname{InfSL}$ from (the opposite of) a category $\mathscr{C}$ with finite products to the category of inf-semilattices, i.e. a contravariant functor $P: \mathscr{C}^{\text {op }} \longrightarrow$ Pos in the category of partial orders Pos such that

- for every object $A$ in $\mathscr{C}$, the partial order $P(A)$ has finite infs

- for every arrow $f: A \rightarrow B$ in $\mathscr{C}$, the monotone map $P_{f}: P(B) \rightarrow P(A)$ preserves them 1

The structure of a primary doctrine is just what is needed to handle a many-sorted logic with binary conjunctions and a true constant, as seen in the following example.

Example 2.2. The leading logical example is the indexed order $L T: \mathscr{V}^{\mathrm{op}} \longrightarrow$ InfSL given by the Lindenbaum-Tarski algebras of well-formed formulae of a first order theory (with only one sort).

Given a theory $\mathcal{T}$ in a first order language $\mathcal{L}$, the domain category of the functor is the category $\mathscr{V}$ of lists of variables and term substitutions:

object of $\mathscr{V}$ are list: 2 of distinct variables $\vec{x}=\left(x_{1}, \ldots, x_{n}\right)$

arrows are lists of substitution 3 for variables $[\vec{t} / \vec{y}]: \vec{x} \rightarrow \vec{y}$ where each term $t_{j}$ in $\vec{t}$ is built in $\mathcal{L}$ on the variables $x_{1}, \ldots, x_{n}$

composition $\vec{x} \stackrel{[\vec{t} / \vec{y}]}{\longrightarrow} \vec{y} \stackrel{[\vec{s} / \vec{z}]}{\longrightarrow} \vec{z}$ is given by simultaneous substitutions

$$
\vec{x} \stackrel{\left[s_{1}[\vec{t} / \vec{y}] / z_{1}, \ldots, s_{k}[\vec{t} / \vec{y}] / z_{k}\right]}{\longrightarrow} \vec{z}
$$

The product of two objects $\vec{x}$ and $\vec{y}$ is given by a(ny) list $\vec{w}$ of as many distinct variables as the sum of the number of variables in $\vec{x}$ and of that in $\vec{y}$. Projections are given by substitution of the variables in $\vec{x}$ with the first in $\vec{w}$ and of the variables in $\vec{y}$ with the last in $\vec{w}$.

\footnotetext{
${ }^{1}$ Here and in the sequel we write the action of a doctrine $P$ on an arrow as $P_{f}$.

${ }^{2}$ The empty list is included.

${ }^{3}$ We shall employ a vector notation for lists of terms in the language as well as for simultaneous substitutions such as $[\vec{t} / \vec{y}]$ in place of $\left[t_{1} / y_{1}, \ldots, t_{m} / y_{m}\right]$. We shall also extend vectorial notation to conjunctions and quantifiers writing $\vec{t}=\vec{s}$ for the conjunction $t_{1}=s_{1} \wedge \ldots \wedge t_{\ell}=s_{\ell}$, provided the lists $\vec{t}$ and $\vec{s}$ are the same length, and writing $\exists_{\vec{x}}$ instead of $\exists_{x_{1}} \ldots \exists_{x_{n}}$.
} 
The functor $L T: \mathscr{V}^{\mathrm{op}} \longrightarrow \operatorname{InfSL}$ is given as follows: for a list of distinct variables $\vec{x}$, the category $\operatorname{LT}(\vec{x})$ has

objects equivalence classes $\mid W\rceil$ of well-formed formulae $W$ of $\mathcal{L}$ with no more free variables than $x_{1}, \ldots, x_{n}$ with respect to provable reciprocal consequence $W \leftarrow_{\mathcal{T}} W^{\prime}$ in $\mathcal{T} 4$

arrows $\mid W\rceil \rightarrow|V|$ are the provable consequences $W \vdash_{\mathcal{T}} V$ in $\mathcal{T}$ for some pair of representatives (hence for any pair)

composition is given by the cut rule in the logical calculus

identities $\lfloor W\rceil \rightarrow\lfloor W\rceil$ are given by the logical rules $W \vdash_{\mathcal{T}} W$

Observe that, in particular, for a list of distinct variables $\vec{x}$, the category $L T(\vec{x})$ has finite limits: products are given by conjunctions of formulae and a terminal object is any provable formula, such as $\vec{x}=\vec{x}$, that is any formula equivalent to the true constant.

Example 2.3. The following example of primary doctrine $S: \mathscr{S}^{\text {op }} \longrightarrow \operatorname{InfSL}$ is the set-theoretic hyperdoctrine described in the introduction and it can be considered in an(y) axiomatic set theory such as ZF. We briefly recall its definition:

- $\mathscr{S}$ is the category of sets and functions,

- $S(A)$ is the poset category of subsets of the set $A$ whose morphisms are inclusions,

- a functor $S_{f}: S(B) \rightarrow S(A)$ acts as the inverse image $f^{-1} U$ on a subset $U$ of the set $B$.

The example 2.2 suggests that, by considering only doctrines, from a logical point of view one restricts attention to the mere existence of a proof of a consequence, i.e. one only deals with proof irrelevance.

As already pointed out in 16, 18, a set-theoretic model of a first order theory determines precisely a functor from the doctrine $L T$ to the doctrine $S$ that preserves all the structure of a primary doctrine.

As the example 2.3, also the example 2.2 gives rise to a Lawvere hyperdoctrine when performed on a many-sorted first order theory giving rise to a cartesian closed base category. And the characterization of set-theoretic model extends directly, see loc.cit.

Remark 2.4. In many senses it is more general - and more elegant - to treat the abstract theory of the relevant structures for the present paper in terms of fibrations. For instance, a different, but equivalent presentation of the structure above is as a faithful fibration $p: \mathscr{A} \rightarrow \mathscr{C}$ between categories with binary products such that $p$ preserves them and has a right inverse right adjoint.

In fact, a primary doctrine $P: \mathscr{C}^{\mathrm{op}} \longrightarrow \operatorname{InfSL}$ determines a faithful fibration $p_{P}: \mathscr{G}_{P} \rightarrow \mathscr{C}$ by a well-known, general construction due to Grothendieck, see [11, 14, which applies to indexed categories. We recall very briefly that

\footnotetext{
${ }^{4}$ We shall denote an equivalence class with representative $x$ as $\lfloor x\rceil$ in order to leave plain square brackets available for other situations.
} 
construction in the present situation. The data for the total category $\mathscr{G}_{P}$ of $P$ are as follows:

objects of $\mathscr{G}_{P}$ are pairs $(A, \alpha)$ such that $A$ is an object in $\mathscr{C}$ and $\alpha$ is an object in $P(A)$.

an arrow $(f, \phi):(A, \alpha) \rightarrow(B, \beta)$ is a pair of an arrow $f: A \rightarrow B$ in $\mathscr{C}$ and an arrow $\phi: \alpha \rightarrow P_{f}(\beta)$

composition of $(f, \phi):(A, \alpha) \rightarrow(B, \beta)$ and $(g, \psi):(B, \beta) \rightarrow(C, \gamma)$ is $(g \circ$ $\left.f, P_{f}(\psi) \circ \phi\right)$.

One checks that a product of objects $(A, \alpha)$ and $(B, \beta)$ is given by

$$
(A, \alpha) \stackrel{\left(\mathrm{pr}_{1}, \pi_{1}\right)}{\longleftrightarrow}(C, \chi) \stackrel{\left(\mathrm{pr}_{2}, \pi_{2}\right)}{\longrightarrow}(B, \beta)
$$

where

$$
A \stackrel{\mathrm{pr}_{1}}{\longleftarrow} \stackrel{\mathrm{pr}_{2}}{\longrightarrow} B
$$

is a product in $\mathscr{C}$ and

$$
P_{\mathrm{pr}_{1}}(\alpha) \stackrel{\pi_{1}}{\longleftarrow} \chi \stackrel{\pi_{2}}{\longrightarrow} P_{\mathrm{pr}_{2}}(\beta)
$$

is a product in $P(C)$.

The first projection extends to a functor $p_{F}: \mathscr{G}_{P} \rightarrow \mathscr{C}$ which is easily seen to be faithful with a right inverse right adjoint.

On the other hand, given a faithful fibration $p: \mathscr{A} \rightarrow \mathscr{C}$, one considers the functor $\widehat{p}: \mathscr{C}^{\text {op }} \longrightarrow \operatorname{InfSL}$ which maps an object $A$ in $\mathscr{C}$ to the partial order which is the poset reflection of the preorder of the vertical arrows on $A$, see 14 , i.e. one first considers the subcategory $p^{A}$ of $\mathscr{A}$ consisting of those objects $\alpha$ such that $p(\alpha)=A$ and a map $g: \alpha \rightarrow \alpha^{\prime}$ of $\mathscr{A}$ is in $p^{A}$ if $p(g)=\mathrm{id}_{A}$; faithfulness of $p$ ensures that the category $p^{A}$ is a preorder. Product preservation ensures that $p^{A}$ has binary products, the right inverse right adjoint ensures that $p^{A}$ has a terminal object. So the poset reflection of $p^{A}$ produces the partial order $\widehat{p}(A)$ on the equivalence classes of objects of $p^{A}$ with respect to the equivalence given by isomorphism, where $\lfloor\alpha\rceil \leq\left\lfloor\alpha^{\prime} \mid\right.$ if there is an arrow $g: \alpha \rightarrow \alpha^{\prime}$ in $p^{A}$ for some pair of representatives (hence for any pair), and the partial order has finite infs.

For an arrow $f: B \rightarrow A$ in $\mathscr{C}$, the functor $\widehat{p}_{f}: \widehat{p}(A) \rightarrow \widehat{p}(B)$ sends an equivalence class $\lfloor\alpha\rceil$ to the equivalence class $\lfloor\beta\rceil$ such that there is a cartesian lifting $g: \beta \rightarrow \alpha$ of $f$.

Setting up an appropriate 2-category for each structure (one for primary doctrines, one for faithful fibrations as above), it is easy to see that the two constructions extend to an equivalence between those 2-categories.

Computing the total category of each of the examples of doctrines in 2.2 and 2.3 , one gets the following.

The total category $\mathscr{G}_{L T}$ is the syntactic presentation of the $\mathcal{L}$-definable subsets of (the finite powers of) a set underlying a model of the theory $\mathcal{T}$ with functions defined by terms in $\mathcal{L}$.

The total category $\mathscr{G}_{S}$ is the full subfibration on subset inclusions of the codomain fibration cod: $\mathscr{S} \rightarrow \longrightarrow \mathscr{S}$ on the category of sets and functions. 
Definition 2.5. A primary doctrine $P: \mathscr{C}^{\mathrm{op}} \longrightarrow$ InfSL is elementary if, for every $A$ and $C$ in $\mathscr{C}$, the functor $P_{i d_{C} \times \Delta_{A}}: P(C \times(A \times A)) \rightarrow P(C \times A) \sqrt{5}$ has a left adjoint $\mathcal{H}_{i d_{C} \times \Delta_{A}}$, and these satisfy

Frobenius reciprocity: for every $A$ and $C$ in $\mathscr{C}$, for $\alpha$ in $P(C \times(A \times A))$, $\beta$ in $P(C \times A)$, the canonical arrow

$$
\exists_{i d_{C} \times \Delta_{A}}\left(P_{i d_{C} \times \Delta_{A}}(\alpha) \wedge_{C \times A} \beta\right) \leq \alpha \wedge_{C \times(A \times A)} \exists_{i d_{C} \times \Delta_{A}}(\beta)
$$

in $P(C \times(A \times A))$ is iso (hence an identity).

Beck-Chevalley condition: for any pullback diagram

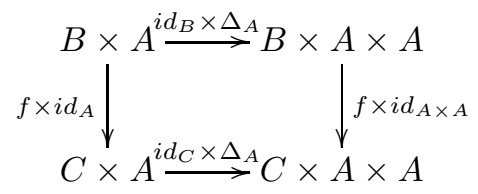

the canonical arrow $\mathcal{H}_{i d_{B} \times \Delta_{A}} P_{f \times i d_{A}}(\alpha) \leq P_{f \times i d_{A \times A}} \mathcal{H}_{i d_{C} \times \Delta_{A}}(\alpha)$ is iso in $P(B \times A \times A)$ for any $\alpha$ in $P(C \times A)$.

We refer the reader to [18, 14 for a thorough analysis of the concepts in the definition just given.

Remark 2.6. For an elementary doctrine $P: \mathscr{C}^{\text {op }} \longrightarrow \operatorname{InfSL}$, for any object $A$ in $\mathscr{C}$, taking $C$ a terminal object, the conditions in 2.5 ensure the existence of a left adjoint $B_{\Delta_{A}}$ to $P_{\Delta_{A}}: P(A \times A) \rightarrow P(A)$. On an object $\alpha$ in $P(A)$ it can be written as

$$
\mathcal{H}_{\Delta_{A}}(\alpha)=P_{\mathrm{pr}_{1}}(\alpha) \wedge_{A \times A} \mathcal{H}_{\Delta_{A}}\left(\top_{A}\right)=P_{\mathrm{pr}_{2}}(\alpha) \wedge_{A \times A} \mathcal{H}_{\Delta_{A}}\left(\top_{A}\right) .
$$

where $\top_{A}$ is the terminal object of $P(A)$.

Because of (1), an abbreviation like $\delta_{A}$ for the object $\exists_{\Delta_{A}}\left(\top_{A}\right)$ is useful.

Example 2.7. For $\mathcal{T}$ a first order theory, the primary fibration $L T: \mathscr{V}^{\mathrm{op}} \longrightarrow$ InfSL, as defined in 2.2, is elementary exactly when $\mathcal{T}$ has an equality predicate.

Example 2.8. The standard example of an elementary doctrine is the fibration of subobjects. Consider a category $\mathscr{X}$ with finite products and pullbacks, i.e. with right adjoints to the diagonal functors $\mathscr{X} \rightarrow \mathscr{X}^{2}$ and $\mathscr{X} \rightarrow \mathscr{X} \rightarrow \downarrow$. The functor $S: \mathscr{X}^{\text {op }} \longrightarrow \operatorname{InfSL}$ assigns to any object $A$ in $\mathscr{X}$ the poset $S(A)$ whose objects are subobjects $\lfloor\alpha: X \gg A\rceil$ in $\mathscr{X}$ with codomain $A$ $\lfloor\alpha\rceil \leq\left\lfloor\alpha^{\prime}\right\rceil$ if there is a commutative diagram

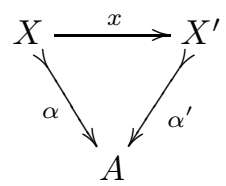

for a (necessarily unique) arrow $x: X \rightarrow X^{\prime}$.

\footnotetext{
${ }^{5}$ We write $\Delta_{A}$ and $f \times f^{\prime}$ respectively for the map $\left\langle\operatorname{id}_{A}, \operatorname{id}_{A}\right\rangle$ and for the map $\left\langle f \circ \operatorname{pr}_{1}, f^{\prime} \circ\right.$ $\left.\operatorname{pr}_{2}\right\rangle: A \times A^{\prime} \rightarrow B \times B^{\prime}$, provided $f: A \rightarrow B$ and $f^{\prime}: A^{\prime} \rightarrow B^{\prime}$.
} 
For an arrow $f: B \rightarrow A$, the assignment mapping an equivalence $[\alpha\rceil$ in $S(A)$ to that represented by the left-hand arrow in the (chosen) pullback

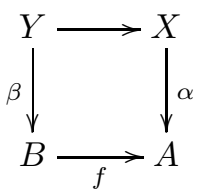

produces a functor $S_{f}: S(A) \rightarrow S(B)$ which preserves products.

Post-composition with an equalizer provides the elementary structure since equalizers are monic.

In example 2.8, we used the same notation for the functor $S$ as in example 2.3 because that is a particular instance of 2.8 when $\mathscr{X}$ is the category $\mathscr{S}$ of sets and functions since each subobject $\lfloor\alpha: X \gg A\rceil$ has a unique inclusion $U \longrightarrow A$ among its representatives.

Example 2.9. Consider a cartesian category $\mathscr{C}$ with weak pullbacks, i.e. for every pair $f: B \rightarrow A, g: C \rightarrow A$ of arrows in $\mathscr{C}$, there is a commutative diagram

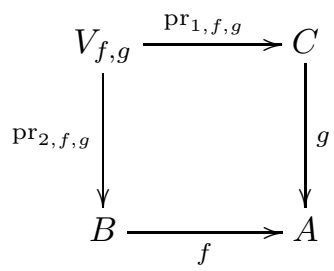

such that, for any $s: T \rightarrow B, t: T \rightarrow C$ satisfying $f \circ s=g \circ t$, there is $u: T \rightarrow V_{f, g}$ such that $t=\operatorname{pr}_{1, f, g} \circ u$ and $s=\operatorname{pr}_{2, f, g} \circ u$.

One can consider the functor $\Psi: \mathscr{C}^{\mathrm{op}} \longrightarrow \operatorname{InfSL}$ given by the poset reflection of each comma category $\mathscr{C} / A$ as $A$ varies over the objects of $\mathscr{C}$. So, for a given object $A$ in $\mathscr{C}$, one first considers the preorder whose

objects are arrows $\alpha: X \rightarrow A$ in $\mathscr{C}$ with codomain $A$

$\alpha \leq \alpha^{\prime}$ if there is an arrow $x: X \rightarrow X^{\prime}$ in $\mathscr{C}$ providing a commutative diagram

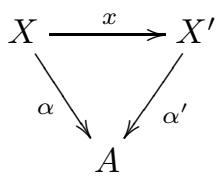

Then quotients that preorder with respect to the reciprocal relation $\alpha \leqslant \alpha^{\prime}$ to obtain the poset $\Psi(A)$.

For an arrow $f: B \rightarrow A$, the assignment that maps an equivalence class $\lfloor\alpha\rceil$ in $\Psi(A)$ to that represented by the left-hand arrow $\operatorname{pr}_{2, f, \alpha}: V_{f, \alpha} \rightarrow B$ in 
the weak (chosen) pullback

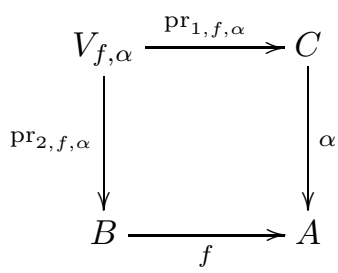

produces a functor $\Psi_{f}: \Psi(A) \rightarrow \Psi(B)$. It is easy to check that it preserves products - which are given by weak pullbacks over $A$.

The elementary structure is given by post-composition with the equalizer.

This example is a slight generalization of a similar one given in [18.

Remark 2.10. Note that the apparently minor difference between the example in 2.8 and that in 2.9 depends crucially on the possibility of factoring an arbitrary arrow as a retraction followed by a monomorphism: for instance, in the category $\mathscr{S}$ of sets and functions, the fact that the two doctrines are equivalent can be achieved thanks to the Axiom of Choice.

Consider the 2-category ED of elementary doctrines:

a 1-arrow from $P$ to $R$ is a pair $(F, b)$ where $F: \mathscr{C} \rightarrow \mathscr{D}$ is a functor which preserves finite products and $b$ is a natural transformation

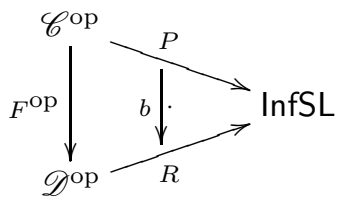

such that for every object $A$ in $\mathscr{C}$, the functor $b_{A}: P(A) \rightarrow R(F(A))$ preserves all the structure. More explicitly, $b_{A}$ preserves finite meets and, for every object $A$ in $\mathscr{C}$,

$$
b_{A \times A}\left(\delta_{A}\right)=R_{\left\langle F\left(\mathrm{pr}_{1}\right), F\left(\mathrm{pr}_{2}\right)\right\rangle}\left(\delta_{F(A)}\right) .
$$

a 2-arrow $\theta:(F, b) \rightarrow(G, c)$ is a natural transformation $\theta: F \rightarrow G$ such that in the diagram

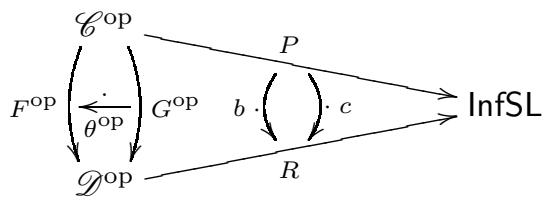

it is $b_{A}(\alpha) \leq R_{\theta_{A}}\left(c_{A}(\alpha)\right)$ for every object $A$ in $\mathscr{C}$ and every $\alpha$ in $P(A)$.

The following definition is similar to 2.5 and contribute the final part of the essential "logical" structure of an indexed poset. 
Definition 2.11. A primary doctrine $P: \mathscr{C}^{\mathrm{op}} \longrightarrow \operatorname{InfSL}$ is existential if, for $A_{1}$ and $A_{2}$ in $\mathscr{C}$, for a(ny) projection pr: $A_{1} \times A_{2} \rightarrow A_{i}, i=1,2$, the functor $P_{\mathrm{pr}_{i}}: P\left(A_{i}\right) \rightarrow P\left(A_{1} \times A_{2}\right)$ has a left adjoint $\mathbb{G}_{\mathrm{pr}_{i}}$, to which we shall unimaginatively refer as existential, and these satisfy

Beck-Chevalley condition: for any pullback diagram

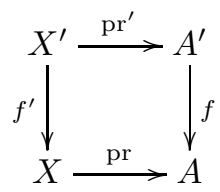

with pr a projection (hence also $\mathrm{pr}^{\prime}$ a projection), for any $\beta$ in $P(X)$, the canonical arrow $\mathcal{G}_{\mathrm{pr}^{\prime}} P_{f^{\prime}}(\beta) \leq P_{f} \mathcal{G}_{\mathrm{pr}}(\beta)$ in $P\left(A^{\prime}\right)$ is iso;

Frobenius reciprocity: for pr: $X \rightarrow A$ a projection, $\alpha$ in $P(A), \beta$ in $P(X)$, the canonical arrow $\mathcal{H}_{\mathrm{pr}}\left(P_{\mathrm{pr}}(\alpha) \wedge_{X} \beta\right) \leq \alpha \wedge_{A} \mathcal{B}_{\mathrm{pr}}(\beta)$ in $P(A)$ is iso.

About this notion we refer the reader to [16, 14].

Examples 2.12. (a) The primary fibration $L T: \mathscr{V}^{\mathrm{op}} \longrightarrow \operatorname{InfSL}$, as defined in 2.2 for a first order theory $\mathcal{T}$, is existential. An existential left adjoint to $P_{\mathrm{pr}}$ is computed by quantifying existentially the variables that are not involved in the substitution given by the projection, e.g. for the projection $\operatorname{pr}=[x / z]:(x, y) \rightarrow(z)$ and a formula $W$ with free variables at most $x$ and $y, \mathcal{H}_{\mathrm{pr}}(W)$ is $\exists_{y}(W[z / x])$.

We stop to note that the example reveals the meaning of the BeckChevalley condition: suppose $S$ and $T$ are sorts and consider the morphism $[x / z]:(x, y) \rightarrow(z)$. On a formula $W$ with free variables at most $x$ and $y$, for any morphism $[t / z]:\left(w_{1}, \ldots, w_{n}\right) \rightarrow(z)$, the diagram

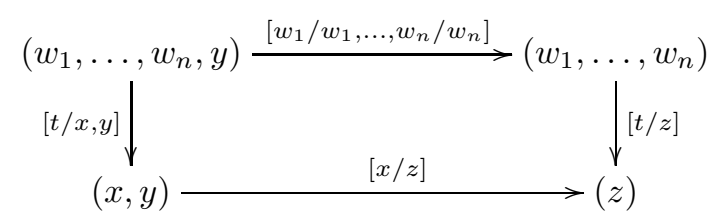

is a pullback and the Beck-Chevalley condition rewrites the fact that substitution commutes with quantification as

$$
\exists_{y}(W[t / x]) \equiv\left(\exists_{y} W[z / x]\right)[t / z]
$$

since the declaration $\left(w_{1}, \ldots, w_{n}\right)$ ensures that $y$ does not appear in $t$.

(b) For a cartesian category $\mathscr{C}$ with weak pullbacks, the elementary doctrine $\Psi: \mathscr{C}^{\mathrm{op}} \longrightarrow \operatorname{InfSL}$ given in 2.9 is existential. Existential left adjoints are given by post-composition.

(c) The primary doctrine in example 2.3 is existential: on a subset $P$ of $A$, the adjoint $\mathcal{H}_{\mathrm{pr}}$, for a projection pr: $A \rightarrow B$, must be evaluated as $\mathcal{H}_{\mathrm{pr}}(P)=$ $\left\{b \in B \mid \exists a \in A\left[a \in \operatorname{pr}^{-1}\{b\} \cap P\right]\right\}$, usually called the image of $P$ along pr. 
Remark 2.13. In an existential elementary doctrine, for every map $f: A \rightarrow B$ in $\mathscr{C}$ the functor $P_{f}$ has a left adjoint $\mathbb{H}_{f}$ that can be computed as

$$
\mathcal{G}_{\mathrm{pr}_{2}}\left(P_{f \times \mathrm{id}_{B}}\left(\delta_{B}\right) \wedge P_{\mathrm{pr}_{1}}(\alpha)\right)
$$

for $\alpha$ in $P(A)$, where $\mathrm{pr}_{1}$ and $\mathrm{pr}_{2}$ are the projections from $A \times B$.

Example 2.14. For a category $\mathscr{X}$ with products and pullbacks, the elementary doctrine $S: \mathscr{X}^{\mathrm{op}} \longrightarrow$ InfSL in 2.8 is existential if and only if $\mathscr{X}$ has a stable proper factorization system $(\mathcal{E}, \mathcal{M})$, see [13, 27]. So, in particular, for $\mathscr{X}$ regular, the subobject doctrine $S: \mathscr{X}^{\mathrm{op}} \longrightarrow \mathrm{InfSL}$ is elementary existential.

Consider the 2-full 2-subcategory EED of ED whose objects are elementary existential doctrines.

The 1-arrows are those pairs $(F, b)$ in ED such that $b$ preserves the left adjoints along projections.

Hence, by 2.13, the second functor of a 1-arrow in EED preserves left adjoints along all arrows in $\mathscr{C}$.

\section{Quotients in an elementary doctrine}

The structure of elementary doctrine is suitable to describe the notion of an equivalence relation and that of a quotient for such a relation.

Definition 3.1. Given an elementary doctrine $P: \mathscr{C}^{\text {op }} \longrightarrow \operatorname{InfSL}$, an object $A$ in $\mathscr{C}$ and an object $\rho$ in $P(A \times A)$, we say that $\rho$ is a $P$-equivalence relation on $A$ if it satisfies

reflexivity: $\delta_{A} \leq \rho$

symmetry: $\rho \leq P_{\left\langle\operatorname{pr}_{2}, \operatorname{pr}_{1}\right\rangle}(\rho)$, for $\operatorname{pr}_{1}, \operatorname{pr}_{2}: A \times A \rightarrow A$ the first and second projection, respectively

transitivity: $P_{\left\langle\mathrm{pr}_{1}, \mathrm{pr}_{2}\right\rangle}(\rho) \wedge P_{\left\langle\mathrm{pr}_{2}, \mathrm{pr}_{3}\right\rangle}(\rho) \leq P_{\left\langle\mathrm{pr}_{1}, \mathrm{pr}_{3}\right\rangle}(\rho)$, for $\mathrm{pr}_{1}, \mathrm{pr}_{2}, \mathrm{pr}_{3}: A \times$ $A \times A \rightarrow A$ the projections to the first, second and third factor, respectively.

Examples 3.2. (a) Given an elementary doctrine $P: \mathscr{C}^{\text {op }} \longrightarrow \operatorname{InfSL}$ and an object $A$ in $\mathscr{C}$, the object $\delta_{A}$ is a $P$-equivalence relation on $A$.

(b) Given a first order theory $\mathcal{T}$ with equality predicate, consider the elementary doctrine $L T: \mathscr{V}^{\mathrm{op}} \longrightarrow$ InfSL as in 2.7. An $L T$-equivalence relation is a $\mathcal{T}$-provable equivalence relation.

(c) For a category $\mathscr{X}$ with products and pullbacks, consider the elementary doctrine of subobjects $S: \mathscr{X}^{\mathrm{op}} \longrightarrow \operatorname{InfSL}$ as in 2.8. An $S$-equivalence relation is an equivalence relation in the category $\mathscr{X}$.

(d) For a cartesian category $\mathscr{C}$ with weak pullbacks, consider the elementary doctrine $\Psi: \mathscr{C}^{\mathrm{op}} \longrightarrow$ InfSL. A $\Psi$-equivalence relation is a pseudo-equivalence relation in $\mathscr{C}$, see 5 .

Remark 3.3. Let $P: \mathscr{C}^{\mathrm{op}} \longrightarrow$ InfSL be an elementary doctrine. For an arrow $f: A \rightarrow B$ in $\mathscr{C}$, the functor $P_{f \times f}: P(B \times B) \rightarrow P(A \times A)$ takes a $P$-equivalence relation $\sigma$ on $B$ to a $P$-equivalence relation on $A$. 
Definition 3.4. Let $P: \mathscr{C}^{\text {op }} \longrightarrow \operatorname{InfSL}$ be an elementary doctrine. Let $\rho$ be a $P$-equivalence relation on $A$.

A quotient of $\rho$ is an arrow $q: A \rightarrow C$ in $\mathscr{C}$ such that $\rho \leq P_{q \times q}\left(\delta_{C}\right)$ and, for every arrow $g: A \rightarrow Z$ such that $\rho \leq P_{g \times g}\left(\delta_{Z}\right)$, there is a unique arrow $h: C \rightarrow Z$ such that $g=h \circ q$. Such a quotient is stable when, for every arrow $f: C^{\prime} \rightarrow C$ in $\mathscr{C}$, there is a pullback

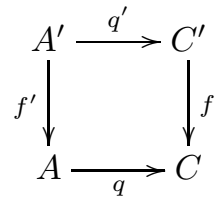

in $\mathscr{C}$ and the arrow $q^{\prime}: A^{\prime} \rightarrow C^{\prime}$ is a quotient of the $P$-equivalence relation $P_{f^{\prime} \times f^{\prime}}(\rho)$.

Let $f: A \rightarrow B$ be an arrow in $\mathscr{C}$. The $P$-kernel of $f: A \rightarrow B$ is the $P$-equivalence relation $P_{f \times f}\left(\delta_{B}\right)$. A quotient $q: A \rightarrow B$ of the $P$-equivalence relation $\rho$ is effective if its $P$-kernel is $\rho$.

Examples 3.5. (a) Given an elementary doctrine $P: \mathscr{C}^{\mathrm{op}} \longrightarrow$ InfSL and an object $A$ in $\mathscr{C}$, a quotient of the $P$-equivalence relation $\delta_{A}$ on $A$ is the identity arrow $\operatorname{id}_{A}: A \rightarrow A$. It is trivially stable and effective by definition.

(b) In the elementary doctrine $S: \mathscr{X}^{\text {op }} \longrightarrow \operatorname{InfSL}$ obtained from a category $\mathscr{X}$ with products and pullbacks, a quotient of the $S$-equivalence relation $\lfloor r: R \longrightarrow A \times A\rceil$ is precisely a coequalizer of the pair of

$$
R \underset{\mathrm{pr}_{2} \circ r}{\stackrel{\mathrm{pr}_{1} \circ r}{\longrightarrow}} A
$$

- hence of any such pair obtained from the class $\lfloor r: R \rightarrow A \times A\rceil$. In particular, all $S$-equivalence relations have quotients which are stable and effective if and only if the category $\mathscr{C}$ is exact.

\section{Set-like doctrines}

We intend to develop doctrines that may interpret constructive theories for mathematics. We shall address two crucial properties that an elementary doctrine should verify in order to sustain such interpretations. One relates to the axiom of comprehension and to equality, the other to quotients.

Definition 4.1. Let $P: \mathscr{C}^{\mathrm{op}} \longrightarrow$ InfSL be a primary doctrine. Let $A$ be an object in $\mathscr{C}$ and $\alpha$ an object in $P(A)$.

A comprehension of $\alpha$ is an arrow $\{\alpha\}: X \rightarrow A$ in $\mathscr{C}$ such that $\top_{X} \leq$ $P_{\{\alpha\}}(\alpha)$ and, for every arrow $g: Y \rightarrow A$ such that $\top_{Y} \leq P_{g}(\alpha)$ there is a unique $h: Y \rightarrow X$ such that $g=\{\alpha\} \circ h$. Such a comprehension is stable when, for every arrow $f: A^{\prime} \rightarrow A$ in $\mathscr{C}, P_{f}(\alpha)$ has a comprehension.

We say that $P: \mathscr{C}^{\text {op }} \longrightarrow \operatorname{InfSL}$ has comprehensions if, for every object $A$ in $\mathscr{C}$, every $\alpha$ in $P(A)$ has a comprehension. 
Again we refer the reader to [18.

The primary doctrine $S: \mathscr{S}^{\mathrm{op}} \longrightarrow$ InfSL of 2.3 has comprehensions given by the trivial remark that a subset determines an actual function by inclusion.

Among the examples listed in 3.2 only example (c), the doctrine of subobjects $S: \mathscr{X}^{\text {op }} \longrightarrow \operatorname{InfSL}$ for $\mathscr{X}$ a category with products and pullbacks, has comprehensions.

Example (d), the elementary doctrine $\Psi: \mathscr{C}^{\text {op }} \longrightarrow$ InfSL constructed as in 2.9 for a cartesian category $\mathscr{C}$ with weak pullbacks, suggests to modify the requirements in 4.1 by dropping uniqueness of the mediating arrows. Before doing that, we note the following.

Remark 4.2. For $f: A^{\prime} \rightarrow A$ in $\mathscr{C}$, the mediating arrow $f^{\prime}$ between the comprehensions $\{\alpha\}: X \rightarrow A$ and $\left\{P_{f}(\alpha)\right\}: X^{\prime} \rightarrow A^{\prime}$ produces a pullback

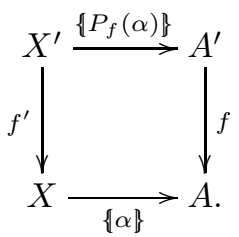

Hence a primary doctrine with comprehensions has comprehensions stable under pullbacks.

Definition 4.3. Let $P: \mathscr{C}^{\text {op }} \longrightarrow \operatorname{InfSL}$ be a primary doctrine. Let $A$ be an object in $\mathscr{C}$ and $\alpha$ an object in $P(A)$.

A weak comprehension of $\alpha$ is an arrow $\{\alpha\}: X \rightarrow A$ in $\mathscr{C}$ such that $\top_{X} \leq P_{\{\alpha\}}(\alpha)$ and, for every arrow $g: Y \rightarrow A$ such that $\top_{Y} \leq P_{g}(\alpha)$ there is a (not necessarily unique) $h: Y \rightarrow X$ such that $g=\{\alpha\} \circ h 6$

Such a comprehension is stable when, for every arrow $f: A^{\prime} \rightarrow A$ in $\mathscr{C}$, $P_{f}(\alpha)$ has a weak comprehension and there is a weak pullback

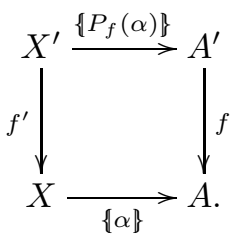

We say that $P: \mathscr{C}^{\text {op }} \longrightarrow$ InfSL, has weak comprehensions if, for every object $A$ in $\mathscr{C}$, every $\alpha$ in $P(A)$ has a weak comprehension.

Remark 4.4. Suppose $\{\alpha\}: X \rightarrow A$ is a weak comprehension of $\alpha$. The arrow $\{\alpha\}$ is monic if and only if it is a strict comprehension.

Thus, when a diagonal is a weak comprehension, the arrow itself satisfies the condition strictly (as in 4.1). But some of its reindexings may satisfy the weaker condition without uniqueness.

\footnotetext{
${ }^{6}$ When necessary to distinguish between the notions in definitions 4.1 and 4.3 we shall refer to one as in 4.1 with the further attribute strict.
} 
Example 4.5. For a cartesian category $\mathscr{C}$ with weak pullbacks, the elementary doctrine $\Psi: \mathscr{C}^{\mathrm{op}} \longrightarrow \operatorname{InfSL}$ as in 2.9 has weak comprehensions.

Proposition 4.6. Suppose $P: \mathscr{C}^{o p} \longrightarrow \mathrm{InfSL}$ is an elementary doctrine. If the diagonal arrow $\Delta_{A}: A \rightarrow A \times A$ is a stable (weak) comprehension of $\delta_{A}$, then for every pair of parallel arrows $X \underset{g}{\stackrel{f}{\rightleftarrows}} A$ in $\mathscr{C}$, the (weak) comprehension of $P_{\langle f, g\rangle}\left(\delta_{A}\right)$ is a (weak) equalizer of $f$ and $g$.

Proof. It follows immediately from the construction of a weak equalizer of $X \underset{g}{\stackrel{f}{\longrightarrow}} A$ as a weak pullback of $\Delta_{A}$ : in the diagram

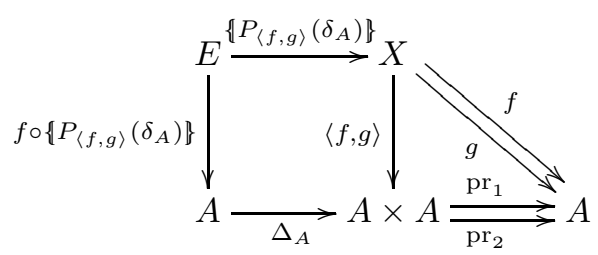

the square is a weak pullback. Since the bottom horizontal arrow is the equalizer of the parallel pair that follows it, the top horizontal arrow is a weak equalizer.

We say that an elementary doctrine $P: \mathscr{C}^{\mathrm{op}} \longrightarrow \mathrm{InfSL}$, has comprehensive (weak) equalizers if, for every object $A$ in $\mathscr{C}$, the diagonal $\Delta_{A}: A \rightarrow A \times A$ is a stable (weak) comprehension of $\delta_{A}$.

Definition 4.7. Let $P: \mathscr{C}^{\text {op }} \longrightarrow \operatorname{InfSL}$ be a primary doctrine. Let $A$ be an object in $\mathscr{C}$ and $\alpha$ an object in $P(A)$.

A (weak) comprehension $\{\alpha\}: X \rightarrow A$ of $\alpha$ is full if $\alpha \leq_{A} \beta$ whenever $\top_{X} \leq_{X} P_{\{\alpha\}}(\beta)$ for $\beta$ in $P(A)$.

Note that the notion of full (weak) comprehension ensures that $\alpha \leq_{A} \beta$ is equivalent to $\top_{X} \leq_{X} P_{\{\alpha\}}(\beta)$ for $\beta$ in $P(A)$.

In an elementary doctrine it follows directly from the definition of $\delta_{A}$ that the diagonal arrow $\Delta_{A}: A \rightarrow A \times A$ is a full comprehension if and only if it is the comprehension of $\delta_{A}$.

Corollary 4.8. Suppose that $P: \mathscr{C}^{o p} \longrightarrow$ InfSL is an elementary doctrine with full comprehensions and comprehensive equalizers. If $f: A \rightarrow B$ is monic, then $P_{f \times f}\left(\delta_{B}\right)=\delta_{A}$.

Proof. It follows by fullness of comprehensions after noting that the comprehension of each side is the kernel of $f$, which in turn follows from 4.6 and the fact that the kernel of a monic is the diagonal.

The next lemma will be needed in section 6 . 
Lemma 4.9. Let $P: \mathscr{C}^{o p} \longrightarrow \mathrm{InfSL}$ be a primary doctrine with full weak comprehensions. Suppose also that, for a given $\alpha$ in $P(A)$, a weak comprehension $\{\alpha\}: X \rightarrow A$ is such that the functor $P_{\{\alpha\}}: P(A) \rightarrow P(X)$ has a right adjoint $\forall_{\{\alpha\}}: P(X) \rightarrow P(A)$. Then $\alpha \wedge-: P(A) \rightarrow P(A)$ has a right adjoint $\alpha \Rightarrow-: P(A) \rightarrow P(A)$.

Proof. Consider $\alpha \Rightarrow \beta:=V_{\{\alpha\}}\left(P_{\{\alpha\}}(\beta)\right)$. To see that $\gamma \leq V_{\{\alpha\}}\left(P_{\{\alpha\}}(\beta)\right)$ if and only if $\alpha \wedge \gamma \leq \beta$ proceed as follows. If $\gamma \leq V_{\{\alpha\}}\left(P_{\{\alpha\}}(\beta)\right)$ then $P_{\{\alpha\}}(\gamma) \leq P_{\{\alpha\}}(\beta)$ and, since a weak comprehension $\{\alpha \wedge \gamma\}: Z \rightarrow A$ of $\alpha \wedge \gamma$ factors through $\{\alpha\}: X \rightarrow A$, then

$$
\top_{Z} \leq P_{\{\alpha \wedge \gamma\}}(\alpha \wedge \gamma) \leq P_{\{\alpha \wedge \gamma\}}(\gamma) \leq P_{\{\alpha \wedge \gamma\}}(\beta)
$$

and, finally full comprehension yields that $\alpha \wedge \gamma \leq \beta$. Next, if $\alpha \wedge \gamma \leq \beta$, then

$$
P_{\{\alpha\}}(\gamma) \leq \top_{X} \wedge P_{\{\alpha\}}(\gamma) \leq P_{\{\alpha\}}(\alpha) \wedge P_{\{\alpha\}}(\gamma) \leq P_{\{\alpha\}}(\alpha \wedge \gamma) \leq P_{\{\alpha\}}(\beta)
$$

Consider the 2-full 2-subcategory EqD of ED whose objects are elementary doctrines with full comprehensions and comprehensive equalizers.

The 1-arrows are those pairs $(F, b)$ in ED such that $F$ preserves comprehensions.

Remark 4.10. The functor $F$ in a pair $(F, b)$ in EqD preserves all finite limits.

The other aspect that we shall consider about set-like doctrines is that every quotient should be of effective descent. We recall the notion of descent data for a $P$-equivalence relation:

Definition 4.11. Given an elementary doctrine $P: \mathscr{C}^{\mathrm{op}} \longrightarrow \operatorname{InfSL}$ and a $P$-equivalence relation $\rho$ on an object $A$ in $\mathscr{C}$, the partial order of descent data $\mathscr{D} e s_{\rho}$ is the sub-order of $P(A)$ on those $\alpha$ such that

$$
P_{\operatorname{pr}_{1}}(\alpha) \wedge_{A \times A} \rho \leq P_{\mathrm{pr}_{2}}(\alpha),
$$

where $\operatorname{pr}_{1}, \operatorname{pr}_{2}: A \times A \rightarrow A$ are the projections.

Remark 4.12. Given an elementary doctrine $P: \mathscr{C}^{\text {op }} \longrightarrow \operatorname{InfSL}$, for $f: A \rightarrow B$ in $\mathscr{C}$, let $\rho$ be the $P$-kernel $P_{f \times f}\left(\delta_{B}\right)$. The functor $P_{f}: P(B) \rightarrow P(A)$ takes values in $\mathscr{D} e s_{\rho} \subseteq P(A)$.

Definition 4.13. Given an elementary doctrine $P: \mathscr{C}^{\mathrm{op}} \longrightarrow \operatorname{InfSL}$ and an arrow $f: A \rightarrow B$ in $\mathscr{C}$, let $\rho$ be the $P$-kernel $P_{f \times f}\left(\delta_{B}\right)$. The arrow $f$ is of effective descent if the functor $P_{f}: P(B) \rightarrow \mathscr{D} e s_{\rho}$ is an isomorphism.

Example 4.14. In the example of the doctrine $S: \mathscr{S}^{\circ \mathrm{op}} \longrightarrow \operatorname{InfSL}$ on the category of sets and functions, as in 2.8 every canonical surjection $f: A \rightarrow A / \sim$, in the quotient of an equivalence relation $\sim$ on $A$, is of effective descent. The condition in 4.13 recognizes the fact that the subsets of the $A / \sim$ are in bijection with those subsets $U$ of $A$ that are closed with respect to the equivalence relation, in the sense that, for $a_{1}, a_{2} \in A$ such that $a_{1} \sim a_{2}$ and $a_{2} \in U$, one has also that $a_{1} \in U$. 
Consider the 2-full 2-subcategory QD of EqD whose objects are elementary doctrines $P: \mathscr{C}^{\text {op }} \longrightarrow$ InfSL in EqD with stable effective quotients of $P$-equivalence relations and of effective descent.

The 1-arrows are those pairs $(F, b)$ in ED such that $F$ preserves quotients and comprehensions.

Proposition 4.15. If $P: \mathscr{C}^{o p} \longrightarrow \mathrm{InfSL}$ is an elementary doctrine in $\mathrm{QD}$, then the category $\mathscr{C}$ is regular. Moreover, if $P: \mathscr{C}^{o p} \longrightarrow$ InfSL is also existential, every equivalence relation in $\mathscr{C}$ has a stable coequalizer.

Proof. The category $\mathscr{C}$ has pullbacks and these can be computed by means of comprehensions: in the case of interest, given an arrow $f: A \rightarrow B$, the comprehension $\{\rho\} \equiv\left\{P_{f \times f}\left(\delta_{B}\right)\right\}: K \rightarrow A \times A$ of the $P$-kernel of $f$ gives a pullback

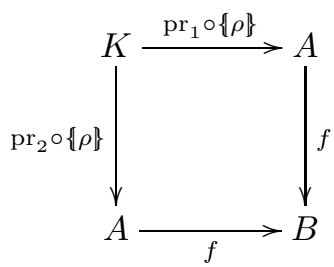

in $\mathscr{C}$ by 4.6. Moreover, thanks to fullness of the comprehension of $\rho$, the quotient $q$ : $A \rightarrow C$ of $\rho$ provides a coequalizer

$$
K \underset{\operatorname{pr}_{2} \circ\{\rho\}}{\stackrel{\operatorname{pr}_{1} \circ\{\rho\}}{\longrightarrow}} A \stackrel{q}{\longrightarrow} C
$$

which is stable thanks to the stability of quotients and comprehensions in $P$. For the second part, suppose that the doctrine $P$ is existential. An equivalence relation $r: R \longrightarrow A \times A$ in $\mathscr{C}$ determines the $P$-equivalence relation $\mathbb{G}_{r}\left(\top_{R}\right)$ on $A$. To conclude note that the mediating arrow $R \rightarrow\left\{\mathcal{H}_{r}\left(\top_{R}\right)\right\}$ is monic.

The following result is a direct consequence of those accomplished in 13, see also [27].

Proposition 4.16. Let $P: \mathscr{C}^{o p} \longrightarrow$ InfSL be a primary doctrine. $P$ is an existential elementary doctrine in QD where every monomorphism in $\mathscr{C}$ is a comprehension if and only if $\mathscr{C}$ is exact and $P$ is equivalent to the doctrine $S: \mathscr{C}^{\circ p} \longrightarrow \operatorname{InfSL}$ of subobjects.

\section{Completing with quotients as a free construction}

There is a fairly obvious construction that produces an elementary doctrine with quotients. We shall present it in the following and prove that it satisfies a universal property. 
Let $P: \mathscr{C}^{\text {op }} \longrightarrow$ InfSL be an elementary doctrine for the rest of the section. Consider the category $\mathscr{Q}_{P}$ of "quotients in $P$ ", the elementary quotient completion of $P$, defined as follows:

an object of $\mathscr{Q}_{P}$ is a pair $(A, \rho)$ such that $\rho$ is a $P$-equivalence relation on A

an arrow $|f|:(A, \rho) \rightarrow(B, \sigma)$ is an equivalence class of arrows $f: A \rightarrow$ $B$ in $\mathscr{C}$ (with a chosen representative) such that $\rho \leq_{A \times A} P_{f \times f}(\sigma)$ in $P(A \times A)$ with respect to the relation determined by the condition that $\rho \leq_{A \times A} P_{f \times g}(\sigma)$

Composition is given by that of $\mathscr{C}$ on representatives, and identities are represented by identities of $\mathscr{C}$.

The indexed partial inf-semilattice $\bar{P}: \mathscr{Q}_{P}{ }^{\text {op }} \longrightarrow$ InfSL on $\mathscr{Q}_{P}$ will be given by categories of descent data: on an object $(A, \rho)$ it is defined as

$$
\bar{P}(A, \rho):=\mathscr{D}_{e} s_{\rho}
$$

and the following lemma is instrumental to give the assignment on arrows by using the action of $P$ on (any) representatives - in the sense that the action of $\bar{P}$ on arrows will then be defined as $\bar{P}_{\lfloor f\rceil}:=P_{f}$ for $\lfloor f\rceil:(A, \rho) \rightarrow(B, \sigma)$.

Lemma 5.1. With the notation used above, let $(A, \rho)$ and $(B, \sigma)$ be objects in $\mathscr{Q}_{P}$, and let $\beta$ be an object in $\mathscr{D} e s_{\sigma}$.

(i) If $f: A \rightarrow B$ is an arrow in $\mathscr{C}$ such that $\rho \leq_{A \times A} P_{f \times f}(\sigma)$, then $P_{f}(\beta)$ is in $\mathscr{D} e s_{\rho}$.

(ii) If $f, g: A \rightarrow B$ are arrows in $\mathscr{C}$ such that $\rho \leq_{A \times A} P_{f \times g}(\sigma)$, then

$$
P_{f}(\beta)=P_{g}(\beta) \text {. }
$$

Proof. (i) is immediate.

(ii) Since $\beta$ is in $\mathscr{D} e s_{\sigma}$, one has that

$$
P_{\mathrm{pr}_{1}^{\prime}}(\beta) \wedge \sigma \leq_{B \times B} P_{\operatorname{pr}_{2}^{\prime}}(\beta)
$$

where $\mathrm{pr}_{1}^{\prime}, \mathrm{pr}_{2}^{\prime}: B \times B \rightarrow B$ are the two projections. Hence

$$
P_{f \times g}\left(P_{\mathrm{pr}_{1}^{\prime}}(\beta)\right) \wedge P_{f \times g}(\sigma) \leq_{A \times A} P_{f \times g}\left(P_{\mathrm{pr}_{2}^{\prime}}(\beta)\right)
$$

since $P_{f \times g}$ preserves the structure. By the hypothesis that $\rho \leq_{A \times A} P_{f \times g}(\sigma)$,

$$
P_{f \circ \mathrm{pr}_{1}}(\beta) \wedge \rho \leq_{A \times A} P_{g \circ \mathrm{pr}_{2}}(\beta)
$$

where $\operatorname{pr}_{1}, \operatorname{pr}_{2}: A \times A \rightarrow A$ are the two projections. Taking $P_{\Delta_{A}}$ of both sides and recalling reflexivity of $\rho$

$P_{f}(\beta)=P_{f}(\beta) \wedge \top_{A}=P_{\Delta_{A}}\left(P_{f \circ \mathrm{pr}_{1}}(\beta)\right) \wedge P_{\Delta_{A}}(\rho) \leq P_{\Delta_{A}}\left(P_{g \circ \mathrm{pr}_{2}}(\beta)\right)=P_{g}(\beta)$.

The other direction follows by symmetry.

Lemma 5.2. With the notation used above, $\bar{P}: \mathscr{Q}_{P}{ }^{o p} \longrightarrow$ InfSL is a primary doctrine. 
Proof. For $(A, \rho)$ and $(B, \sigma)$ in $\mathscr{Q}_{P}$ let $\operatorname{pr}_{1}, \operatorname{pr}_{3}: A \times B \times A \times B \rightarrow A$ and $\mathrm{pr}_{2}, \mathrm{pr}_{4}: A \times B \times A \times B \rightarrow B$ be the four projections. The meet of two $P$-equivalence relations on $A \times B$

$$
\rho \bigotimes \sigma:=P_{\left\langle\mathrm{pr}_{1}, \mathrm{pr}_{3}\right\rangle}(\rho) \wedge_{A \times B \times A \times B} P_{\left\langle\mathrm{pr}_{2}, \mathrm{pr}_{4}\right\rangle}(\sigma)
$$

is a $P$-equivalence relation on $A \times B$ and it provides an object $(A \times B, \rho \bigotimes \sigma)$ which, together with the arrows determined by the two projections from $A \times B$, gives a product of $(A, \rho)$ and $(B, \sigma)$ in $\mathscr{Q}_{P}$.

For each $(A, \rho)$, the sub-partial order $\mathscr{D} e s_{\rho} \subseteq P(A)$ is closed under finite meets.

Assume that $P$ has weak comprehensions for the rest of the section.

Lemma 5.3. With the notation used above, $\bar{P}$ is an elementary doctrine with comprehensions and comprehensive equalizers. If $P$ has full weak comprehensions, then $\bar{P}$ has full comprehensions.

Proof. First we show that $\mathscr{Q}_{P}$ has equalizers, hence all finite limits. Consider a parallel pair $\lfloor f\rceil,\lfloor g\rceil:(A, \rho) \rightarrow(B, \sigma)$, and let $e:=\left\{P_{\langle f, g\rangle}(\sigma)\right\}: E \rightarrow A$ be a weak comprehension. It is easy to see that $\mid e\rceil:\left(E, P_{e \times e}(\rho)\right) \rightarrow(A, \rho)$ is an equalizer as required.

A similar argument shows that $\bar{P}$ has comprehensions. More precisely, given $(A, \rho)$ and $\beta$ in $\mathscr{D} e s_{\rho}$, let $\{\beta\}: X \rightarrow A$ be a weak comprehension for $\beta$ over $A$ in the doctrine $P$. A comprehension for $\beta$ over $(A, \rho)$ in $\bar{P}$ is

$$
\lfloor\{\beta\}\rceil:\left(X, P_{\{\beta\} \times\{\beta\}}(\rho)\right) \rightarrow(A, \rho) .
$$

Fullness of weak comprehensions in $P$ implies fullness of comprehensions in $\bar{P}$ because objects of $\bar{P}$ on $(A, \rho)$ are descent data related to $P$.

A left adjoint $\overline{\mathcal{H}}_{\left[\Delta_{A}\right\rceil}$ for $\bar{P}_{\left[\Delta_{A}\right\rceil}$ is computed by

$$
\overline{\bar{H}}_{\left[\Delta_{A}\right\rceil}(\alpha):=P_{\operatorname{pr}_{1}}(\alpha) \wedge_{A \times A} \rho
$$

for $\alpha$ in $\mathscr{D} e s_{\rho}$. Indeed, let $\theta$ be in $\mathscr{D} e s_{\rho \otimes \rho}$ such that $\alpha \leq_{(A, \rho)} \bar{P}_{\left.\mid \Delta_{A}\right\rceil}(\theta)$, i.e. $\alpha \leq_{A} P_{\Delta_{A}}(\theta)$. Thus $\mathbb{H}_{\Delta_{A}}(\alpha) \leq_{A \times A} \theta$ and one has by remark 2.6

$$
\begin{aligned}
P_{\mathrm{pr}_{1}^{\prime}}(\alpha) \wedge P_{\left\langle\mathrm{pr}_{1}^{\prime}, \mathrm{pr}_{2}^{\prime}\right\rangle}\left(\delta_{A}\right) \wedge P_{\left\langle\mathrm{pr}_{2}^{\prime}, \mathrm{pr}_{3}^{\prime}\right\rangle}(\rho) & \leq A \times A \times A \\
& P_{\langle\times A \times A} P_{\left\langle\mathrm{pr}_{1}^{\prime}, \mathrm{pr}_{2}^{\prime}\right\rangle}(\theta) \wedge P_{\left\langle\mathrm{pr}_{2}^{\prime}, \mathrm{pr}_{3}^{\prime}\right\rangle}(\theta)
\end{aligned}
$$

for $\operatorname{pr}_{i}^{\prime}: A \times A \times A \rightarrow A, i=1,2,3$, the three projections. Hence $P_{\mathrm{pr}_{1}}(\alpha) \wedge$ $\rho \leq_{A \times A} \theta$, which gives $\overline{\mathcal{H}}_{\left\lfloor\Delta_{A}\right\rceil}(\alpha) \leq_{(A \times A, \rho \bigotimes \rho)} \theta$. It is easy to prove the converse that, if $\overline{\mathcal{H}}_{\left\lfloor\Delta_{A}\right\rceil}(\alpha) \leq \theta$, then $\alpha \leq \bar{P}_{\left\lfloor\Delta_{A}\right\rceil}(\theta)$.

More generally, a left adjoint $\overline{\mathcal{G}}_{\left[i d_{C} \times \Delta_{A}\right\rceil}$ for $\bar{P}_{\left[i d_{C} \times \Delta_{A}\right\rceil}$ is computed analogously by

$$
\overline{\bar{G}}_{\left[i d_{C} \times \Delta_{A}\right]}(\alpha):=P_{\left\langle\mathrm{pr}_{1}, \mathrm{pr}_{2}\right\rangle}(\alpha) \wedge_{C \times A \times A} P_{\left\langle\mathrm{pr}_{2}, \mathrm{pr}_{3}\right\rangle}(\rho)
$$


for $\alpha$ in $\bar{P}((C, \sigma) \times(A, \rho))$ with $\operatorname{pr}_{1}: C \times A \times A \rightarrow C$ and $\operatorname{pr}_{i}: C \times A \times A \rightarrow A$ for $i=2,3$ the corresponding projections.

Finally, $\bar{P}$ has comprehensive equalizers by construction.

A careful reader may have noticed that the hypothesis on weak comprehensions in $P$ in 5.3 was needed in order to construct all pullbacks and characterize regular monos in $\mathscr{Q}_{P}$.

Lemma 5.4. With the notation used above, $\bar{P}$ has effective quotients of $\bar{P}$ equivalence relations and those are of effective descent.

Proof. Since the sub-partial order $\mathscr{D} e s_{\rho} \subseteq P(A)$ is closed under finite meets, a $\bar{P}$-equivalence relation $\tau$ on $(A, \rho)$ is also a $P$-equivalence relation on $A$. It is easy to see that $\left[\operatorname{id}_{A}\right\rceil:(A, \rho) \rightarrow(A, \tau)$ is an effective quotient and of effective descent since $\rho \leq_{A \times A} \tau$.

Lemma 5.5. With the notation used above, a quotient of $\bar{P}$-equivalence is stable.

Proof. Let $\tau$ be a $\bar{P}$-equivalence relation on $(A, \rho)$, let $\left\lfloor\operatorname{id}_{A}\right\rceil:(A, \rho) \rightarrow(A, \tau)$ be its quotient, and let $\lfloor f\rceil:(B, \sigma) \rightarrow(A, \tau)$ be an arrow in $\mathscr{Q}_{P}$. By the previous lemma 5.3, in $\mathscr{Q}_{P}$ there is a pullback diagram

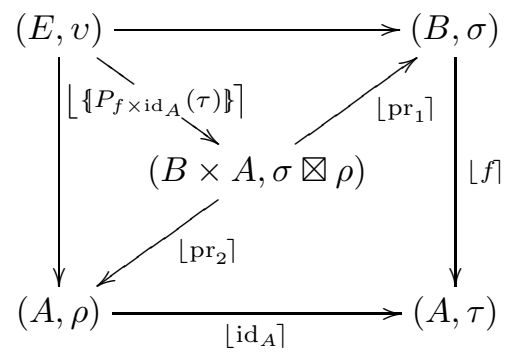

where $v:=P_{\left\{P_{f \times \mathrm{id}_{A}}(\tau)\right\} \times\left\{P_{f \times \mathrm{id}_{A}}(\tau)\right\}}(\sigma \otimes \rho)$. Let $e:=\left\{P_{f \times \mathrm{id}_{A}}(\tau)\right\}$ and $\omega:=$ $P_{\text {exe }}(\sigma \otimes \tau)$. The arrow $\left\langle\operatorname{id}_{B}, f\right\rangle: B \rightarrow B \times A$ of $\mathscr{C}$ factors through the comprehension $\left\{P_{f \times \mathrm{id}_{A}}(\tau)\right\}: E \rightarrow B \times A$ because

$$
\top_{B} \leq P_{f}\left(P_{\Delta_{A}}(\tau)\right)=P_{\langle f, f\rangle}(\tau)=P_{\left\langle\operatorname{id}_{B}, f\right\rangle}\left(P_{f \times \operatorname{id}_{A}}(\tau)\right)
$$

say $\left\langle\operatorname{id}_{B}, f\right\rangle=\left\{P_{f \times \operatorname{id}_{A}}(\tau)\right\} \circ g=e \circ g$. Moreover

$$
\sigma \leq P_{\left\langle\operatorname{id}_{B}, f\right\rangle \times\left\langle\operatorname{id}_{B}, f\right\rangle}(\sigma \otimes \tau)=P_{g \times g}\left(P_{e \times e}(\sigma \otimes \tau)\right)=P_{g \times g}(\omega)
$$

hence producing a commutative diagram

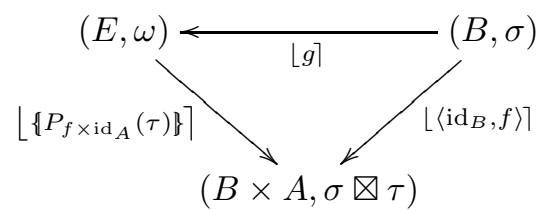

in $\mathscr{Q}_{P}$. It is easy to see that $\lfloor g\rceil:(B, \sigma) \rightarrow(E, \omega)$ has a retraction

$$
\left[\operatorname{pr}_{1} \circ\left\{P_{f \times \operatorname{id}_{A}}(\tau)\right\} \mid:(E, \omega) \rightarrow(B, \sigma)\right.
$$


which is monic and hence an inverse of $\lfloor g\rceil$. Therefore $(B, \sigma)$ becomes a quotient of $\omega$ on $(E, v)$.

There is a 1-arrow $(J, j): P \rightarrow \bar{P}$ in ED. The functor $J: \mathscr{C} \rightarrow \mathscr{Q}_{P}$ sends an object $A$ in $\mathscr{C}$ to $\left(A, \delta_{A}\right)$ and an arrow $f: A \rightarrow B$ to $\lfloor f\rceil:\left(A, \delta_{A}\right) \rightarrow\left(B, \delta_{B}\right)$ since $\delta_{A} \leq_{A \times A} P_{f \times f}\left(\delta_{B}\right)$ by [3.2(a) and 3.3. The functor $J$ is full.

For $A$ in $\mathscr{C}$, the partial order $\bar{P}\left(A, \delta_{A}\right)=\mathscr{D} e s_{\delta_{A}}$ is $P(A)$ since $P_{\mathrm{pr}_{1}} \alpha \wedge$ $\delta_{A} \leq_{A \times A} P_{\mathrm{pr}_{2}} \alpha$ for any $\alpha$ in $P(A)$. Take the function $j_{A}: P(A) \rightarrow \bar{P}\left(A, \delta_{A}\right)$ to be the identity.

Lemma 5.6. With the notation used above,

(i) every object in $\mathscr{Q}_{P}$ is a quotient of a $\bar{P}$-equivalence relation on an object in the image of $J$

(ii) every object in the image of $J$ is projective with respect to quotients of $\bar{P}$-equivalence relation.

Proof. (i) The object $(A, \rho)$ is a quotient of $\left(A, \delta_{A}\right)$.

(ii) A quotient of a $\bar{P}$-equivalence relation is isomorphic to one that has an identity as a representative.

Assume that $P$ has comprehensive weak equalizers for the rest of the section.

Lemma 5.7. With the notation used above, the functor $J: \mathscr{C} \rightarrow \mathscr{Q}_{P}$ is faithful.

Proof. For $f, g: A \rightarrow B$ suppose that $J(f)=J(g)$. In other words,

$$
\mathbb{B}_{\Delta_{A}}\left(\top_{A}\right)=\delta_{A} \leq_{A \times A} P_{f \times g}\left(\delta_{B}\right)
$$

and equivalently $\top_{A} \leq_{A} P_{\Delta_{A}}\left(P_{f \times g}\left(\delta_{B}\right)\right)={ }_{A} P_{\langle f, g\rangle}\left(\delta_{B}\right)$ since $\mathcal{H}_{\Delta_{A}} \dashv P_{\Delta_{A}}$. It follows that the identity on $A$ equalizes $f$ and $g$, thanks to lemma 4.6 and the hypothesis that $P$ has comprehensive weak equalizers.

The doctrine $P: \mathscr{C}^{\mathrm{op}} \longrightarrow \operatorname{InfSL}$ in 5.7 is obtained from $\bar{P}: \mathscr{Q}_{P}{ }^{\mathrm{op}} \longrightarrow$ InfSL by change of base along $J$ since $j$ is the identity natural transformation. In logical terms, this states that the theory expressing $P$ extended with a quotient constructor is conservative over the original theory.

We can now prove that the assignment $P \mapsto \bar{P}$ gives a left bi-adjoint to the forgetful 2-functor $U: \mathrm{QD} \rightarrow \mathrm{EqD}$.

Theorem 5.8. For every elementary doctrine $P: \mathscr{C}^{o p} \longrightarrow \operatorname{InfSL}$ in EqD precomposition with the 1-arrow

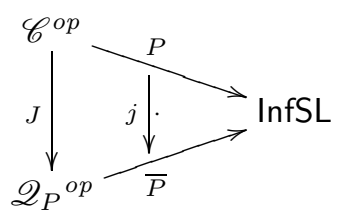

in $\mathrm{EqD}$ induces an essential equivalence of categories

$$
\text { - }(J, j): \mathrm{QD}(\bar{P}, X) \equiv \operatorname{EqD}(P, X)
$$

for every $X$ in QD. 
Proof. Suppose $X$ is a doctrine in QD. As to full faithfulness of the functor in (3), consider two pairs $(F, b)$ and $(G, c)$ of 1 -arrows from $\bar{P}$ to $X$. By 5.6 , the natural transformation $\theta: F \rightarrow G$ in a 2 -arrow from $(F, b)$ to $(G, c)$ in QD is completely determined by its action on objects in the image of $J$. And, since a quotient $q: U \rightarrow V$ of an $X$-equivalence relation on $U$ is of effective descent, $X(V)$ is a full sub-partial order of $X(U)$. Essential surjectivity of the functor in (3) follows from 5.6. Preservation of comprehensions for $J$ follows from 4.8 because a comprehension is monic.

The reason why $-\circ(J, j)$ need not be a strong equivalence in 5.8 is that quotients in the doctrine $X$ are determined only up to iso. Since quotients in the doctrine $\bar{P}$ are determined by a specified construction, it is possible to strengthen the result by restricting the 2-categories QD and EqD to doctrines with a choice of the categorical structure.

Examples 5.9. (a) The structure of the elementary quotient completion for the doctrine $L T: \mathscr{V}^{\mathrm{op}} \longrightarrow \operatorname{InfSL}$ from a first order theory $\mathcal{T}$ with an equality predicate depends heavily on the choice of basic operation symbols used to present the theory $\mathcal{T}$, see 4.9 and 6.7

(b) For a cartesian category $\mathscr{C}$ with weak pullbacks, the elementary quotient completion of the elementary doctrine $\Psi: \mathscr{C}^{\mathrm{op}} \longrightarrow \operatorname{InfSL}$ given in 2.9 is essentially equivalent to the exact completion of $\mathscr{C}$, see [7].

Remark 5.10. By 4.15, the elementary quotient completion $\mathscr{Q}_{S}$ of an elementary doctrine $S: \mathscr{X}^{\mathrm{op}} \longrightarrow \mathrm{InfSL}$ in EqD, obtained from a category $\mathscr{X}$ with products and pullbacks as in 2.8, is regular. Then, it may seem natural to compare $\mathscr{Q}_{S}$ with the regular completion of $\mathscr{X}$, see [4, or with the exact/regular completion of $\mathscr{X}$ in case $\mathscr{X}$ is regular, see [10, 4]. The three constructions are in general not equivalent; that will be treated in future work. Also, an example of an elementary quotient completion that is not exact will be presented in section 7.2 .

In case an elementary doctrine $P: \mathscr{C}^{\mathrm{op}} \longrightarrow \operatorname{InfSL}$ with comprehensive equalizers fails to have all comprehensions, it is possible to add these freely (hence fully) preserving the rest of the structure by taking the fibration of vertical maps on the total category of $P$, see [14, 30].

\section{Applications to richer doctrines}

We shall now analyse the extent to which the elementary quotient completion construction produced in the previous section behaves with respect to further logical structure. We start with existential doctrines.

Theorem 6.1. Suppose P: $\mathscr{C}^{o p} \longrightarrow$ InfSL is an existential elementary doctrine. Then the doctrine $\bar{P}: \mathscr{Q}_{P}{ }^{o p} \longrightarrow$ InfSL is existential and the pair $(J, j): P \rightarrow \bar{P}$ is a 1-arrow in EED.

Proof. The left adjoint along a projection with respect to $\bar{P}$ is given by the left adjoint along the underlying projection in $\mathscr{C}$ with respect to $P$. 
We now consider other logical structures such as implications and universal quantification, always remaining within Lawvere's unifying schema of "logical constructs as adjoints", see [16, 14.

Definition 6.2. A primary doctrine $P: \mathscr{C}^{\mathrm{op}} \longrightarrow \operatorname{InfSL}$ is implicational if, for every object $A$ in $\mathscr{C}$, every $\alpha$ in $P(A)$, the functor $\alpha \wedge-: P(A) \rightarrow P(A)$ has a right adjoint $\alpha \Rightarrow-: P(A) \rightarrow P(A)$.

Examples 6.3. (a) The primary fibration $L T: \mathscr{V}^{\mathrm{op}} \longrightarrow \operatorname{InfSL}$, as defined in 2.2 for a first order theory $\mathcal{T}$, is implicational since, for $V$ and $W$ in $L T(\vec{x})$, the formula $V \Rightarrow W$ gives the value of the right adjoint of $V \wedge-$ on $W$.

(b) The primary doctrine in example 2.3 is implicational: on a subset $P$ of $A$, the right adjoint $P \Rightarrow-$ on a subset $Q$ of $A$ is computed as

$$
P \Rightarrow Q:=\{a \in A \mid a \in P \Rightarrow a \in Q\}
$$

Definition 6.4. A primary doctrine $P: \mathscr{C}^{\text {op }} \longrightarrow \operatorname{InfSL}$ is universal if, for $A_{1}$ and $A_{2}$ in $\mathscr{C}$, for a(ny) projection $\operatorname{pr}_{i}: A_{1} \times A_{2} \rightarrow A_{i}, i=1,2$, the functor $P_{\mathrm{pr}_{i}}: P\left(A_{i}\right) \rightarrow P\left(A_{1} \times A_{2}\right)$ has a right adjoint $V_{\mathrm{pr}_{i}}$, and these satisfy the Beck-Chevalley condition:

for any pullback diagram

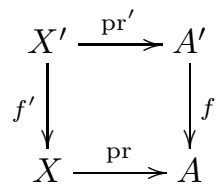

with pr a projection (hence also $\mathrm{pr}^{\prime}$ a projection), for any $\beta$ in $P(X)$, the canonical arrow $P_{f} V_{\mathrm{pr}}(\beta) \leq{V_{\mathrm{pr}}} P_{f^{\prime}}(\beta)$ in $P\left(A^{\prime}\right)$ is iso;

Examples 6.5. (a) The primary fibration $L T: \mathscr{V}^{\mathrm{op}} \longrightarrow \operatorname{InfSL}$, as defined in 2.2 for a first order theory $\mathcal{T}$, is universal. A right adjoint to $P_{\mathrm{pr}}$ is computed by quantifying universally the variables that are not involved in the substitution given by the projection, e.g. for the projection $\mathrm{pr}=[z / x]:(x, y) \rightarrow(z)$ and a formula $W$ with free variables at most $x$ and $y, \forall_{\mathrm{pr}}(W)$ is $\forall_{y}(W[z / x])$. As in the case of the left adjoints in 2.11, the Beck-Chevalley condition expresses the correct interplay between term substitution and universal quantification.

(b) The primary doctrine in example2.3 is universal: on a subset $U$ of $A$, the adjoint $V_{\mathrm{pr}}$, for a projection pr, is given by $V_{\mathrm{pr}}(U):=\left\{b \in B \mid \operatorname{pr}^{-1}\{b\} \subseteq U\right\}$.

Remark 6.6. In an elementary doctrine $P: \mathscr{C}^{\text {op }} \longrightarrow$ InfSL which is implicational and universal for every map $f: A \rightarrow B$ in $\mathscr{C}$ the functor $P_{f}$ has a right adjoint $V_{f}$ that can be computed as

$$
V_{\mathrm{pr}_{2}}\left(P_{f \times \mathrm{id}_{B}}\left(\delta_{B}\right) \Rightarrow P_{\mathrm{pr}_{1}}(\alpha)\right)
$$

for $\alpha$ in $P(A)$, where $\operatorname{pr}_{1}$ and $\operatorname{pr}_{2}$ are the projections from $A \times B$ - similarly to 2.13 .

Moreover, if $P$ has full weak comprehensions, then the converse also holds by 4.9 . 
The following is an instance of a general situation for the quotient completion: a weak representation in the doctrine $P$ gives rise to a strict representation in the completion $\bar{P}$. Recall from [6] that a weak evaluation from $A$ to $B$ is an arrow $w:\left[B^{A}\right] \times A \rightarrow B$ such that for every arrow $f: X \times A \rightarrow B$ there is an arrow $f^{\prime}: X \rightarrow\left[B^{A}\right]$ with $f=w \circ\left(f^{\prime} \times \operatorname{id}_{A}\right)$. In loc.cit., the notion above was instrumental to give a characterization of those exact completions which are locally cartesian closed; it helps to show how the more general structure in the present paper extends that of exact completion, see also [31. We follow [6] and say that $\mathscr{C}$ is weakly cartesian closed if it has a weak evaluation for each pair of objects.

Proposition 6.7. Let $P: \mathscr{C}^{o p} \longrightarrow$ InfSL be an elementary, implicational, universal doctrine with weak comprehensions. If $\mathscr{C}$ is weakly cartesian closed, then $\mathscr{Q}_{P}$ is cartesian closed and $\bar{P}$ is implicational and universal. Moreover, the 1 -arrow $(J, j): P \rightarrow \bar{P}$ preserves the implicational and universal structure.

Proof. Suppose that $(A, \rho)$ and $(B, \sigma)$ are objects in $\mathscr{Q}_{P}$ and $w:\left[B^{A}\right] \times A \rightarrow$ $B$ is a weak evaluation from $A$ to $B$ in $\mathscr{C}$. Recalling 4.9, consider the object $\varphi$ of $P\left(\left[B^{A}\right] \times\left[B^{A}\right]\right)$ :

$$
\varphi=V_{\mathrm{pr}_{3}} V_{\mathrm{pr}_{4}}\left(P_{\left\langle\mathrm{pr}_{3}, \mathrm{pr}_{4}\right\rangle}(\rho) \Rightarrow P_{\left\langle w\left\langle\mathrm{pr}_{1}, \mathrm{pr}_{3}\right\rangle, w\left\langle\mathrm{pr}_{2}, \mathrm{pr}_{4}\right\rangle\right\rangle}(\sigma)\right)
$$

where $\operatorname{pr}_{1}, \mathrm{pr}_{2}:\left[B^{A}\right] \times\left[B^{A}\right] \times A \times A \rightarrow\left[B^{A}\right]$ and $\mathrm{pr}_{3}, \mathrm{pr}_{4}:\left[B^{A}\right] \times\left[B^{A}\right] \times$ $A \times A \rightarrow A$ are the projections. Within the logic provided by the doctrine, it can be described as the intension of those pairs $\left(e_{1}, e_{2}\right)$ of "elements of $\left[B^{A}\right]$ " such that

$$
\forall_{a_{3} \in A} \forall_{a_{4} \in A}\left(a_{3} \rho a_{4} \Rightarrow w\left(e_{1}, a_{3}\right) \sigma w\left(e_{2}, a_{4}\right)\right)
$$

where we have used the same indices for the variables as those for the projections. It is easy to see that $\varphi$ satisfies the conditions of symmetry and transitivity in 3.1 so that, considered a weak comprehension $\left\{P_{\left.\Delta_{[B} A\right]}(\varphi)\right\}: F \rightarrow$ $\left[B^{A}\right]$ of $P_{\Delta_{\left[B^{A}\right]}}(\varphi)$ in $P\left(\left[B^{A}\right]\right)$, the object $\left.\psi:=P_{\left\{P_{\left.\Delta_{[B} A\right]}\right.}(\varphi)\right\} \times\left\{P_{\left.\Delta_{[B} A\right]}(\varphi)\right\}(\varphi)$ is a $P$-equivalence relation. It is straightforward to prove that

$$
w \circ\left(\left\{P_{\left.\Delta_{[B} A\right]}(\varphi)\right\} \times \operatorname{id}_{A}\right):(F, \psi) \times(A, \rho) \rightarrow(B, \sigma)
$$

is a (strict) evalutation from $(A, \rho)$ to $(B, \sigma)$.

The right adjoints required for the implicational and universal structures on $\bar{P}$ are computed by the corresponding right adjoints with respect to $P$.

As an application, we shall consider the Axiom of Unique Choice (AUC) within an existential doctrine $P: \mathscr{C}^{\text {op }} \longrightarrow \operatorname{InfSL}$ with the same structure as in 6.7. Recall that (AUC) states that, in a cartesian closed regular category, a total relation, which is also single-valued, contains the graph of a function, see e.g. 29. We shall adapt it to the more general frame of existential doctrines as in 6.7. Let $w:\left[B^{A}\right] \times A \rightarrow B$ be a weak evaluation from $A$ to $B$ in $\mathscr{C}$. We 
say that (AUC) from $A$ to $B$ holds for $w$ if, for every $\rho$ in $P(A \times B)$,

$$
\begin{aligned}
& \underset{A \rightarrow 1}{\forall} \underset{A \times B \rightarrow A}{\stackrel{⿴}{H}}(\rho) \wedge \\
& \wedge \underset{B \times B \rightarrow 1}{\forall} \underset{A \times B \times B \rightarrow B \times B}{\forall}\left(\left(P_{\left\langle\mathrm{pr}_{1}, \mathrm{pr}_{2}\right\rangle}(\rho) \wedge P_{\left\langle\mathrm{pr}_{1}, \mathrm{pr}_{3}\right\rangle}(\rho)\right) \Rightarrow P_{\left\langle\mathrm{pr}_{2}, \mathrm{pr}_{3}\right\rangle}\left(\delta_{B}\right)\right) \leq
\end{aligned}
$$

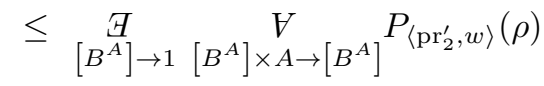

where we dispensed with the labels on the various appropriate projection arrows as these are easily reconstructed.

Using plain logical notation (as in the proof of 6.7),

$$
\begin{aligned}
& \forall_{x_{1} \in A} \exists_{y_{2} \in B} x_{1} \rho y_{2} \wedge \\
& \wedge \forall_{y_{2} \in B} \forall_{y_{3} \in B} \forall_{x_{1} \in A}\left(\left(x_{1} \rho y_{2} \wedge x_{1} \rho y_{3}\right) \Rightarrow y_{2} \delta_{B} y_{3} \leq\right. \\
& \leq \exists_{h_{1} \in\left[B^{A}\right]} \forall_{x_{2} \in A} x_{2} \rho w\left(h_{1}, x_{2}\right) .
\end{aligned}
$$

We skip the easy proof that, if (AUC) from $A$ to $B$ holds for a weak evaluation, then it holds for every weak evaluation. For that reason, from now on we say that (AUC) from $A$ to $B$ holds.

Corollary 6.8. Suppose that $P: \mathscr{C}^{o p} \longrightarrow$ InfSL is an elementary existential, implicational and universal doctrine with weak comprehensions. Suppose that $\mathscr{C}$ is weakly cartesian closed and that $A$ and $B$ are objects in $\mathscr{C}$. The following are equivalent:

(i) in $P$ (AUC) from $A$ to $B$ holds

(ii) in $\bar{P}$ (AUC) from $\left(A, \delta_{A}\right)$ to $\left(B, \delta_{B}\right)$ holds.

Proof. It follows directly from the preservation properties of the 1-arrow $(J, j): P \rightarrow \bar{P}$ by proposition 6.1 and 6.7 .

\section{Examples from constructive foundations}

The main applications of the elementary quotient completion appear in the formalization of constructive mathematics in type theory. As we already explained, the ability of constructing quotients of equivalence relations is an essential tool of standard mathematics; any formalization of constructive mathematics that intends to achieve foundational relevance must allow treatment of such quotients in some way, in particular if it is inherently intensional. We shall sketch two examples of elementary quotient completion in type theory: one is given over intensional Martin-Löf's type theory [25] and the other over the intensional level of the minimalist foundation [21. Both models are based on total setoids à la Bishop [3, with proof-terms as morphisms.

\subsection{The setoid model over Martin-Löf's Type Theory}

The model of "setoids" over intensional Martin-Löf's Type Theory, see [26, 9], is an instance of the exact completion of a category with finite products and weak equalizers. We already know that it is an elementary quotient completion, so we simply review the elementary doctrine one can construct in order to obtain it as such a completion.

The base category is a syntactic construction $\mathscr{M} \mathscr{L}$ is defined as follows. 
The objects of $\mathscr{M} \mathscr{L}$ are closed sets of MLTT.

An arrow $\lfloor t \in B[x \in A]]: A \rightarrow B$ is an equivalence class of terms $t \in$ $B[x \in A] 7$ derivable in MLTT where two terms $t \in B[x \in A]$ and $t^{\prime} \in B[x \in A]$ are equivalent if there is a proof-term in MLTT

$$
p \in \operatorname{Id}\left(B, t, t^{\prime}\right)[x \in A]
$$

The composition of $\lfloor t \in B[x \in A]]: A \rightarrow B$ and $\lfloor s(x) \in C[x \in B]]$ is defined as

$$
\lfloor s(t) \in C[x \in A]]: A \rightarrow C .
$$

The identity morphism on $A$ is $[x \in A[x \in A]]: A \rightarrow A$.

Lemma 7.1. The category $\mathscr{M} \mathscr{L}$ has finite products and weak equalizers.

Proof. A terminal object is the singleton $N_{1}$. A product of closed sets $A, B$ is $\sum_{x \in A} B$ with its first and second projections since

$$
\text { idpeel }(z,(x, y) \cdot \operatorname{id}(\langle x, y\rangle)) \in \operatorname{Id}\left(\sum_{x \in A} B,\left\langle\pi_{1}(z), \pi_{2}(z)\right\rangle, z\right)
$$

thanks to the $\beta$-rules.

A weak equalizer of $\lfloor t \in B[x \in A]],\left\lfloor t^{\prime} \in B[x \in A]\right]: A \rightarrow B$ is

$$
\left\lfloor\pi_{1}(z) \in A\left[z \in \sum_{x \in A} \operatorname{ld}\left(B, t, t^{\prime}\right)\right]\right]: \sum_{x \in A} \operatorname{ld}\left(B, t, t^{\prime}\right) \rightarrow A
$$

The functor $F^{M L}: \mathscr{M} \mathscr{L}^{\text {op }} \longrightarrow \operatorname{InfSL}$ is defined on a closed set $A$ as the partial order consisting of

equivalence classes $\mid \phi$ prop $[x \in A] \mid$ of predicates in MLTT depending on $A$ with respect to equiprovability, i.e. $\phi \operatorname{prop}[x \in A] \sim \phi^{\prime} \operatorname{prop}[x \in A]$ if there is a proof of $\phi \leftrightarrow \phi^{\prime} \operatorname{prop}[x \in A]$ in MLTT,

$\lfloor\phi \operatorname{prop}[x \in A] \mid \leq\lfloor\psi \operatorname{prop}[x \in A]]$ if there is a proof-term $r \in \psi[x \in$ $A, w \in \phi]$ in MLTT.

The action of the functor on arrows in $\mathscr{M} \mathscr{L}$ is given by substitution.

Proposition 7.2. The doctrine $F^{M L}: \mathscr{M} \mathscr{L}^{o p} \longrightarrow$ InfSL is existential elementary, with full weak comprehensions and comprehensive weak equalizers.

Proof. Products of propositions and the singleton set provide finite meets thanks to the identification of propositions with sets typical of MLTT. The left adjoint to substitution with $\mid t(x) \in B[x \in A]]: A \rightarrow B$ is computed, for $\phi(x) \operatorname{prop}[x \in A]$, as the equivalence class represented by the proposition

$$
\sum_{x \in A}(\operatorname{ld}(B, t(x), y) \wedge \phi(x)) \operatorname{prop}[y \in B] .
$$

A weak comprehension $\{\phi\}$ of $\phi \operatorname{prop}[x \in A]$ is given by

$$
\left\lfloor\pi_{1}(w) \in A\left[w \in \sum_{x \in A} \phi\right]\right]: \sum_{x \in A} \phi \rightarrow A .
$$

Note that weak comprehensions are full: it is enough to show a proof of $\phi \leq \exists_{\{\phi\}} \top_{W}$ (with $W$ codomain of $\{\phi\}$ ) given by

$$
\langle\langle y, p\rangle,\langle\operatorname{id}(y), *\rangle\rangle \in \sum_{w \in W}\left(\operatorname{Id}\left(A, \pi_{1}(w), y\right) \wedge N_{1}\right)[y \in A, p \in \phi] .
$$

\footnotetext{
${ }^{7}$ To represent a term depending on a variable $x$, we shall indifferently use the metavariable $t$ or $t(x)$. We use the latter when we need to refer to a substitution of a variable $x$ that may appear in $t$.
} 
with $*$ the canonical element of the singleton set $N_{1}$. It follows by an immediate, direct calculation that weak equalizers are comprehensions.

The doctrine $F^{M L}: \mathscr{M} \mathscr{L}^{\text {op }} \longrightarrow$ InfSL enjoys more properties.

Proposition 7.3. The category $\mathscr{M} \mathscr{L}$ is weakly cartesian closed. For every arrow $f$ in $\mathscr{C}$, the functor $F_{f}^{M L}$ has also a right adjoint (which necessarily satisfies Beck-Chevalley condition).

Proof. The category $\mathscr{M} \mathscr{L}$ is weakly cartesian closed because given sets $A$ and $B$, a weak evaluation is defined via the dependent product as

$$
\left\lfloor\pi_{1}(z)\left(\pi_{2}(z)\right) \in B\left[z \in\left(\prod_{x \in A} B\right) \times A\right]\right]:\left(\prod_{x \in A} B\right) \times A \rightarrow B .
$$

It is only a weak evalutation because inhabitation of $\operatorname{ld}(B(x), f(x), g(x))[x \in$ $A]$ cannot ensure inhabitation of $\operatorname{Id}\left(\prod_{x \in A} B(x), \lambda x . f(x), \lambda x . g(x)\right)$, see [12]. A right adjoint to substitution is given by the dependent product set.

In fact, the doctrine $F^{M L}$ is isomorphic to the doctrine $\Psi^{\mathscr{M} \mathscr{L}}: \mathscr{M} \mathscr{L}^{\text {op }} \longrightarrow$ InfSL, constructed as in 2.9 from the category $\mathscr{M} \mathscr{L}$. The isomorphism

$$
(\operatorname{Id} \mathscr{M} \mathscr{L}, h): F^{M L} \rightarrow \Psi^{\mathscr{M} \mathscr{L}}
$$

has the identity functor in the first component and, for $A$ a closed set, the functor $h(A): F^{M L}(A) \rightarrow \Psi^{\mathscr{M} \mathscr{L}}(A)$ maps $\mid \phi(x)$ prop $[x \in A] \mid$ to the equivalence class represented by the arrow

$$
\left\lfloor\pi_{1}(w) \in A\left[w \in \sum_{x \in A} \phi(x)\right]\right]: \sum_{x \in A} \phi(x) \rightarrow A
$$

which has codomain $A$. Note that a proof-term $t(y) \in \psi[x \in A, y \in \phi]$ produces a map and a commutative triangle

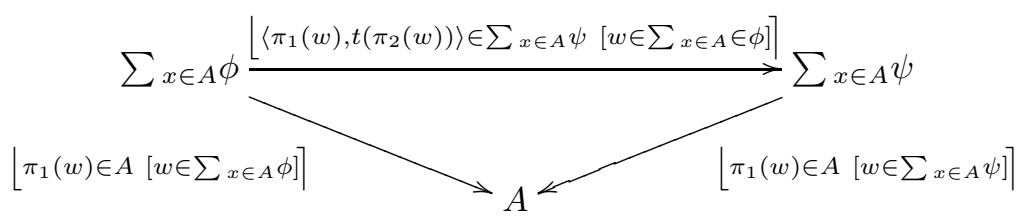

which shows that the assignment for $h(A)$ given in (4) does not depend on the choice of representatives and it extends to a functor. Moreover, one can easily check that the functor $k(A): \Psi^{\mathscr{M} \mathscr{L}}(A) \rightarrow F^{M L}(A)$ mapping $f(y) \in A[y \in B]$ to $\sum_{y \in B} \operatorname{ld}(A, f(y), x)$ for $x \in A$ provides an inverse to $h(A)$.

Remark 7.4. The base category $\mathscr{Q}_{F^{M L}}$ of the elementary quotient completion $\overline{F^{M L}}$ of $F^{M L}: \mathscr{M} \mathscr{L}^{\text {op }} \longrightarrow$ InfSL is essentially equivalent to the model of total setoids over Martin-Löf type theory as given in [26]. 


\subsection{The quotient completion in the two-level minimalist foundation}

One of the motivations for developing the notion of elementary quotient completion is to describe abstractly the "quotient model" given in [21. That model is built over a dependent type theory acting as the intensional level of the two-level minimalist foundation for constructive mathematics in 21]. It is used to give an interpretation of the extensional level of the foundationdenoted emtt for extensional minimal type theory - in the intensional leveldenoted mtt for minimal type theory. Quite similar to that built over MartinLöf's type theory, also this model is based on the idea of total setoids à la Bishop, but it appears to have different properties from that. For instance, the setoid model built over a set-theoretic fragment of mtt is not exact as we note in the following - hence it cannot be an exact completion.

Recall from 21 that mtt is a predicative version of Coquand's Calculus of Constructions $\mathrm{CoC}$, see [8. Indeed, as $\mathrm{CoC}$, it resembles a many sorted logic with propositions given primitively and depending on sorts. Propositions are equipped with proof-terms and sorts include dependent sets as in MartinLöf's type theory. But, contrary to CoC, in mtt there are two types of sorts on which propositions depend: these are sets and collections, where the first are included in the latter; this distinction resembles the usual distinction between sets and classes in NBG and is instrumental to represent the power collection of a set which, from a predicative point of view, need not be a set. However the whole mtt can be interpreted in $\mathrm{CoC}$ if one interprets both sets and collections in $\mathbf{m t t}$ as sets in CoC. Indeed, if in mtt collections were identified with sets then one would just get $\mathrm{CoC}$. Therefore, many properties of the quotient model over mtt can be extended to the corresponding quotient model over CoC.

Corresponding to sets and collections in mtt we consider two doctrines: one is a sub-doctrine of the other. The larger has the base category formed by collections with their typed terms, the other has the base category restricted to the full subcategory on sets.

The "syntactic category of collections in mtt" $\mathcal{C M}$ is constructed like the previous example. It has closed collections of mtt as objects. An arrow $\lfloor t \in B[x \in A]]: A \rightarrow B$ from the closed collection $A$ to the closed collection $B$ is an equivalence class of proof-terms in mtt where $t \in A[x \in B]$ is equivalent to $t^{\prime} \in A[x \in B]$ if there is a proof-term

$$
p \in \operatorname{Id}\left(A, t, t^{\prime}\right)[x \in B]
$$

in mtt. The composition of the two arrows $\lfloor t \in B[x \in A]]: A \rightarrow B$ and $\lfloor s(y) \in C[y \in B]]: B \rightarrow C$ is given as the equivalence class

$$
\lfloor s(t) \in C[x \in A]]: A \rightarrow C
$$

with identity arrows given by $\mid x \in A[x \in A]]: A \rightarrow A$.

The "syntactic category of sets in $\mathbf{m t t}$ " $\mathcal{S M}$ is the full subcategory of $\mathcal{C} \mathcal{M}$ on the closed sets.

Like in the previous example, the two categories just introduced have weak limits. 
Lemma 7.5. The categories $\mathcal{C} \mathcal{M}$ and $\mathcal{S} \mathcal{M}$ are cartesian and with weak equalizers.

Proof. The proof is analogous to that of 7.1 .

We now define a doctrine on each syntactic category defined on mtt in a similar way to that for Martin-Löf's type theory.

The functor

$$
G^{\text {mtt }}: \mathcal{C} \mathcal{M}^{\text {op }} \longrightarrow \operatorname{InfSI}
$$

is defined on a closed collection $A$ as the partial order consisting of

equivalence classes $|\phi \operatorname{prop}[x \in A]|$ of predicates in mtt depending on $A$ with respect to equiprovability, i.e. $\phi \operatorname{prop}[x \in A] \sim \phi^{\prime} \operatorname{prop}[x \in A]$ if there is a proof of $\phi \leftrightarrow \phi^{\prime}$ prop $[x \in A]$ in $\mathbf{m t t}$,

$\lfloor\phi \operatorname{prop}[x \in A]] \leq\lfloor\psi \operatorname{prop}[x \in A]]$ if there is a proof-term $r \in \psi[x \in$ $A, w \in \phi]$ in mtt.

The action of the functor on arrows in $\mathcal{C M}$ is given by substitution.

The doctrine

$$
F^{\text {mtt }}: \mathcal{S} \mathcal{M}^{\text {op }} \longrightarrow \operatorname{InfSI}
$$

is defined on a closed set $A$ as the partial order consisting of

equivalence classes $\left\lfloor\phi \operatorname{prop}_{s}[x \in A]\right]$ of small propositions $\phi(x) \operatorname{prop}_{s}[x \in$

$A]$ depending on $A$, i.e. propositions closed only under quantification on sets, with respect to equiprovability in mtt,

$\left\lfloor\phi \operatorname{prop}_{s}[x \in A]\right] \leq\left\lfloor\phi^{\prime} \operatorname{prop}_{s}[x \in A] \mid\right.$ if there is a proof-term of $r \in \phi^{\prime}[x \in$ $A, w \in \phi]$ in mtt.

The action of the functor on arrows in $\mathcal{S M}$ is given by substitution.

Proposition 7.6. The doctrines $G^{\text {mtt }}: \mathcal{C} \mathcal{M}^{o p} \longrightarrow \operatorname{InfSI}$ and $F^{\text {mtt }}: \mathcal{S} \mathcal{M}^{o p} \longrightarrow$ InfSI are existential elementary, implicational and universal, with full weak comprehensions and comprehensive weak equalizers. Moreover, the doctrine $F^{\text {mtt }}$ is weakly cartesian closed.

Proof. The proof for each is analogous to that for Martin-Löf's type theory in propositions 7.2 and 7.3 .

Remark 7.7. Similarly to 7.4, there is an essential equivalence between the completion $\overline{G^{\text {mtt }}}$ of the doctrine $G^{\text {mtt }}$ and the model of extensional collections in [21, and there is another between the completion $\overline{F^{\text {mtt }}}$ of the doctrine $F^{\mathbf{m t t}}$ and the model of extensional sets in [21].

Then, if one recalls that emtt is the extensional version of mtt with the addition of effective quotients, proof-irrelevance of propositions and extensional equality of functions, it is easy to see that there is an obvious interpretation of emtt in the doctrine $\overline{G^{\mathbf{m t t}}}$ provided that a choice of the structure is given.

Actually, by means of logical constructors of emtt, following the paradigm proposed in [16, 14, we can describe the logical theories modelled by most of the doctrines considered in this paper. 
For example, the notion of elementary existential doctrine with full comprehensions and comprehensive equalizers is the necessary structure to interpret the set-theoretic fragment of emtt where sets just include the singleton set, binary products and indexed sums of a set with a proposition, and whose propositions are just closed under conjunctions, extensional equality, existential quantifiers and the truth constant. Then the extension with emttquotients captures the logical theory behind existential doctrines in QD.

Remark 7.8. The quotient model $\mathscr{Q}_{F \text { mtt }}$ of $F^{\text {mtt }}$ for the fragment of mtt in 22] provides a genuine example that the elementary quotient completion is not an exact completion.

Indeed, consider that in an exact, locally cartesian closed category, (AUC) always holds, as shown in [20]. Now, if $\mathscr{Q}_{F^{\mathrm{mtt}}}$ were exact, then in the original doctrine $F^{\text {mtt }}$ (AUC) from any object to any other would hold by 6.8 . But 22 shows that (AUC) from the natural numbers to the natural numbers does not hold.

Knowing that in $\mathrm{CoC}$ (AUC) from an object to another does not hold in general, see [29], with the same argument as above we get another example of elementary quotient completion that is not exact: take $\mathscr{Q}_{F^{\mathrm{CoC}}}$ with $F^{\mathrm{CoC}}$ defined as $F^{\mathbf{m t t}}$ but within CoC.

\section{Conclusions and future work}

The notion of elementary quotient completion developed in the previous sections was inspired by the need to give an abstract presentation of the quotient model used in 21. This notion is more general than that of exact completion. As remarked in 5.10, an exact completion of a cartesian category with weak pullbacks is an instance of the elementary quotient completion construction. On the other hand, as remarked in 7.8, there are elementary quotient completions which are not exact.

Relevant instances of elementary quotient completion are used to formalize mathematics within an intensional type theory. They are applied to turn a theory with intensional and weak structures into one with extensional and strong ones. For example, the category $\mathscr{Q}_{F \mathbf{m t t}}$ of extensional sets over the intensional theory $\mathbf{m t t}$, as well as the category $\mathscr{Q}_{F^{M L}}$ of setoids over Martin-Löf's type theory, are cartesian closed, while the corresponding categories of sets of $\mathbf{m t t}$ and of Martin-Löf's type theory are not. Moreover the doctrines $F^{M L}, F^{\mathbf{m t t}}$ and $G^{\mathbf{m t t}}$ associated to $\mathbf{m t t}$ and to Martin-Löf's type theory satisfy only weak comprehension, because their propositions may be equipped with more than one proof.

But it is worth mentioning that to view an elementary quotient completion, as that over mtt in [21], as a model of an extensional theory as emtt in 21, one needs to solve a coherence problem. Indeed, the base category of an elementary quotient completion, as well as of an exact completion in [5], does not provide an explicit construction for the categorical structure used to interpret logical sorts. 
In 21] we solved the problem by using an ad hoc coherence theorem to select the model structure necessary to interpret the syntax of emtt in the elementary quotient completion over mtt. Since that theorem heavily relies on the fact that the syntax of $\mathbf{m t t}$ is defined inductively, a possible direction of further investigation is to explore how to interpret a logical theory corresponding to doctrines in QD in (the doctrine $\bar{P}$ of) an elementary quotient completion of a doctrine $P$ in EqD.

In future work we shall investigate the case of completing an existential elementary doctrine with respect to quotients with a more relaxed notion of arrow and compare it with the elementary quotient completion introduced here.

Acknowledgements. The authors would like to acknowledge Giovanni Sambin and Ruggero Pagnan for very many, useful and intensive discussions on the subject. Discussions with Fabio Pasquali and Lorenzo Malatesta were also very useful for the second author.

\section{References}

[1] G. Barthes, V. Capretta, and O. Pons. Setoids in type theory. J. Funct. Programming, 13(2):261-293, 2003.

[2] L. Birkedal, A. Carboni, G. Rosolini, and D.S. Scott. Type theory via exact categories. In V. Pratt, editor, Proc. 13th Symposium in Logic in Computer Science, pages 188-198, Indianapolis, 1998. IEEE Computer Society.

[3] E. Bishop. Foundations of Constructive Analysis. McGraw-Hill Book Co., 1967.

[4] A. Carboni. Some free constructions in realizability and proof theory. J. Pure Appl. Alg., 103:117-148, 1995.

[5] A. Carboni and R. Celia Magno. The free exact category on a left exact one. J. Austr. Math. Soc., 33(A):295-301, 1982.

[6] A. Carboni and G. Rosolini. Locally cartesian closed exact completions. J. Pure Appl. Alg., 154:103-116, 2000.

[7] A. Carboni and E.M. Vitale. Regular and exact completions. J. Pure Appl. Alg., 125:79-117, 1998.

[8] T. Coquand. Metamathematical investigation of a calculus of constructions. In P. Odifreddi, editor, Logic in Computer Science, pages 91-122. Academic Press, 1990.

[9] P. Dybjer. Internal type theory. In TYPES '95, volume 1158 of Lecture Notes in Computer Science, pages 120-134. Springer-Verlag, 1996.

[10] P.J. Freyd and A. Scedrov. Categories Allegories. North Holland Publishing Co., 1991.

[11] A. Grothendieck. Catégories fibrées et descent (Exposé VI). In A. Grothendieck, editor, Revêtements etales et groupe fondamental - SGA 1, number 224 in Lecture Notes in Mathematics, pages 145-194. Springer-Verlag, 1971.

[12] M. Hofmann. Extensional Constructs in Intensional Type Theory. Distinguished Dissertations. Springer-Verlag, 1997. 
[13] J. Hughes and B. Jacobs. Factorization systems and fibrations: Toward a fibred Birkhoff variety theorem. Electron. Notes Theor. Comp. Sci., 11:\#, 2002.

[14] B. Jacobs. Categorical Logic and Type Theory. North-Holland Publishing Co., 1999.

[15] J. Lambek and P.J. Scott. Introduction to higher order categorical logic. Cambridge University Press, 1986.

[16] F.W. Lawvere. Adjointness in foundations. Dialectica, 23:281-296, 1969.

[17] F.W. Lawvere. Diagonal arguments and cartesian closed categories. In Category Theory, Homology Theory and their Applications, II (Battelle Institute Conference, Seattle, Wash., 1968, Vol. Two), pages 134-145. Springer-Verlag, 1969.

[18] F.W. Lawvere. Equality in hyperdoctrines and comprehension schema as an adjoint functor. In A. Heller, editor, Proc. New York Symposium on Application of Categorical Algebra, pages 1-14. Amer.Math.Soc., 1970.

[19] F.W. Lawvere and R. Rosebrugh. Sets for Mathematics. Cambridge University Press, 2003.

[20] M.E. Maietti. Modular correspondence between dependent type theories and categories including pretopoi and topoi. Math. Structures Comp. Sci., 15(6):1089-1149, 2005.

[21] M.E. Maietti. A minimalist two-level foundation for constructive mathematics. Ann. Pure Appl. Logic, 160(3):319-354, 2009.

[22] M.E. Maietti. Consistency of the minimalist foundation with Church thesis and Bar Induction. submitted, 2010.

[23] M.E. Maietti and G. Sambin. Toward a minimalist foundation for constructive mathematics. In L. Crosilla and P. Schuster, editors, From Sets and Types to Topology and Analysis: Practicable Foundations for Constructive Mathematics, number 48 in Oxford Logic Guides, pages 91-114. Oxford University Press, 2005.

[24] M. Makkai and G. Reyes. First Order Categorical Logic, volume 611 of Lecture Notes in Mathematics. Springer-Verlag, 1977.

[25] B. Nordström, K. Petersson, and J. Smith. Programming in Martin Löf's Type Theory. Clarendon Press, Oxford, 1990.

[26] E. Palmgren. Bishop's set theory. Slides for a lecture at the TYPES summer school, 2005.

[27] D. Pavlović. Maps II: chasing proofs in the Lambek-Lawvere logic. Log. J. IGPL, 4(2):159-194, 1996.

[28] G. Sambin and S. Valentini. Building up a toolbox for Martin-Löf's type theory: subset theory. In G. Sambin and J. Smith, editors, Twenty-five years of constructive type theory, pages 221-244. Oxford University Press, 1998.

[29] Th. Streicher. Semantics of type theory. Birkhäuser, 1991.

[30] P. Taylor. Practical Foundations of Mathematics. Cambridge University Press, 1999.

[31] J. van Oosten. Realizability: An Introduction to its Categorical Side, volume 152. North-Holland Publishing Co., 2008. 
Quotient completion for the foundation of constructive mathematics 33

\author{
Maria Emilia Maietti \\ Dipartimento di Matematica Pura ed Applicata \\ Università di Padova \\ via Trieste 63 \\ 35121 Padova, Italy \\ e-mail: maietti@math.unipd.it \\ Giuseppe Rosolini \\ DIMA, Università di Genova \\ via Dodecaneso 35 \\ 16146 Genova, Italy \\ e-mail: rosolini@unige.it
}

